\title{
Effective Lagrangian for a light Higgs-like scalar
}

\author{
Roberto Contino, ${ }^{a}$ Margherita Ghezzi, ${ }^{a}$ Christophe Grojean, ${ }^{b, c}$ Margarete Mühlleitner ${ }^{d}$ \\ and Michael Spira ${ }^{e}$ \\ ${ }^{a}$ Dipartimento di Fisica, Università di Roma La Sapienza and INFN, Roma, Italy \\ ${ }^{b}$ ICREA at IFAE, Universitat Autònoma de Barcelona, E-08193 Bellaterra, Spain \\ ${ }^{c}$ Theory Division, Physics Department, CERN, Geneva, Switzerland \\ ${ }^{d}$ Institute for Theoretical Physics, Karlsruhe Institute of Technology, Karlsruhe, Germany \\ ${ }^{e}$ Paul Scherrer Institut, CH-5232 Villigen PSI, Switzerland \\ E-mail: roberto.contino@roma1.infn.it, \\ margherita.ghezzi@roma1.infn.it, christophe.grojean@cern.ch, \\ milada.muehlleitner@kit.edu, michael.spira@psi.ch
}

ABstRACT: We reconsider the effective Lagrangian that describes a light Higgs-like boson and better clarify a few issues which were not exhaustively addressed in the previous literature. In particular we highlight the strategy to determine whether the dynamics responsible for the electroweak symmetry breaking is weakly or strongly interacting. We also discuss how the effective Lagrangian can be implemented into automatic tools for the calculation of Higgs decay rates and production cross sections.

Keywords: Higgs Physics, Beyond Standard Model, Technicolor and Composite Models

ARXIV EPRINT: 1303.3876 


\section{Contents}

1 Introduction 1

2 Effective Lagrangian for a light Higgs doublet 3

2.1 Current bounds on flavor-preserving operators 7

3 Estimates of the effects on physics observables 10

3.1 Operators sensitive to a strongly-interacting Higgs boson 10

3.2 Operators sensitive to the scale of New Physics 12

$\begin{array}{ll}3.3 & \text { Operators generated at the one-loop level } \\ 3.4\end{array}$

$\begin{array}{lll}3.4 & \text { Fermionic operators } & 16\end{array}$

$\begin{array}{ll}\text { 3.5 Non-linear Lagrangian for a Higgs-like scalar } & 17\end{array}$

$\begin{array}{lll}3.6 & \text { Implications of custodial symmetry } & 18\end{array}$

4 Implementing the Higgs effective Lagrangian beyond the tree level 20

$\begin{array}{lll}\text { 4.1 RG evolution of the Wilson coefficients } & 21\end{array}$

4.2 Decay rates at the loop level with the effective Lagrangian 23

$\begin{array}{llr}5 & \text { Discussion } & 28\end{array}$

$\begin{array}{ll}\text { A SM Lagrangian: notations and conventions } & 31\end{array}$

B Electroweak Chiral Lagrangian in non-unitary gauge 32

C Relaxing the CP-even hypothesis $\quad 34$

D Current bounds on dimension-6 operators 35

\section{Introduction}

The exploration of the weak scale has marked an important step forward with the discovery by the ATLAS [1] and CMS [2] collaborations of a boson with mass $m_{h} \simeq 125 \mathrm{GeV}$, whose production cross section and decay rates are compatible with those predicted for the Higgs boson of the Standard Model (SM). At the same time, no hint of the existence of additional new particles has emerged yet, which might shed light on the origin of the electroweak symmetry breaking (EWSB). One is thus faced with the problem of which is the best strategy to describe the properties and investigate the nature of the new boson $h$, beyond the framework of the Standard Model. In absence of a direct observation of new states, our ignorance of the EWSB sector can be parametrized in terms of an effective Lagrangian for the light boson. Such an effective description is valid as long as New Physics 
(NP) states appear at a scale $M \gg m_{h}$, and is based on an expansion in the number of fields and derivatives [3]. The detailed form of the effective Lagrangian depends on which assumptions are made. Considering that the observation made by the LHC experiments is in remarkable agreement with the SM prediction, although within the current limited experimental precision, it is reasonable to assume that $h$ is a CP-even scalar that forms an $\mathrm{SU}(2)_{L}$ doublet together with the longitudinal polarizations of the $W$ and $Z$, so that the $\mathrm{SU}(2)_{L} \times \mathrm{U}(1)_{Y}$ electroweak symmetry is linearly realized at high energies. Under these assumptions the effective Lagrangian can be expanded into a sum of operators with increasing dimensionality, where the leading NP effects are given by dimension- 6 operators.

The parametrization of the deviations of the Higgs couplings in terms of higherdimension operators started more than two decades ago. The experimental observation of the Higgs boson, however, calls for a more detailed analysis. First, a compilation of a complete and updated list of constraints on the various Wilson coefficients is in need. Second, the rather precise estimation of the Higgs mass below the gauge boson thresholds necessitates a careful computation including off-shell effects that have not been incorporated up-to-now when the SM Lagrangian is supplemented by higher-dimensional operators. It is the purpose of this paper to perform such an updated analysis. We will also discuss in detail the implications of the custodial symmetry on the generalized Higgs couplings and clarify a few other issues which were not exhaustively addressed in the previous literature, like for example the connection with the effective Lagrangian for a non-linearly realized electroweak symmetry. Finally, a precise comparison of the Higgs couplings with the SM predictions can only be done when higher-order effects are included in a consistent way, and we will develop a strategy to this end.

The paper is structured as follows. In section 2 we review the construction of the effective Lagrangian for a light Higgs doublet. By means of a naive power counting we estimate the coefficients of the various operators and review the most important bounds set on them by present experimental results on electroweak (EW) and flavor observables. Focusing on Higgs physics, we then discuss in section 3 the relative effect of the various operators on physical observables. Such an analysis, first proposed in ref. [4], will allow us to identify which operators can probe the Higgs coupling strength to the new states and which instead are sensitive only to the mass scale $M$. This is of key importance to distinguish between weakly-coupled UV completions of the Standard Model, like Supersymmetric (SUSY) theories, and theories where the EW symmetry is broken by a new strongly-interacting dynamics which forms the Higgs boson as a bound state [4-13]. These are the two most compelling scenarios put forward to solve the hierarchy problem of the Standard Model. We conclude the section by discussing how the assumption of a Higgs doublet and linearly-realized $\mathrm{SU}(2)_{L} \times \mathrm{U}(1)_{Y}$ can be relaxed. We illustrate the non-linear effective Lagrangian valid for the case of a generic CP-even scalar $h$ and discuss the implications of custodial invariance. Section 4 is devoted to clarify a few issues related to the use of the effective Lagrangian beyond the tree level. We present our concluding discussion in section 5. In the appendices $\mathrm{A}-\mathrm{C}$ we collect useful formulas and give further details on the construction of the effective Lagrangian. The details of how we derived the bounds on the dimension- 6 operators are reported in appendix D. 
As an illustration of our analysis and to better demonstrate how the effective Lagrangian can be implemented into automatic tools for the computation of physical quantities like Higgs production cross sections and decay rates, we have written eHDECAY, ${ }^{1}$ a modified version of the program HDECAY [14, 15], which includes the full list of leading bosonic operators. We will describe the program in a separate companion paper [16].

\section{Effective Lagrangian for a light Higgs doublet}

The most general $\mathrm{SU}(3)_{C} \times \mathrm{SU}(2)_{L} \times \mathrm{U}(1)_{Y}$-invariant Lagrangian for a weak doublet $H$ at the level of dimension- 6 operators was first classified in a systematic way in refs. [17-19]. Subsequent analyses [20-25] pointed out the presence of some redundant operators, and a minimal and complete list of operators was finally provided in ref. [26]. As recently discussed in ref. [4], a convenient basis of operators relevant for Higgs physics, assuming that the Higgs is a CP-even weak doublet (this assumption will be relaxed in appendix C) and the baryon and lepton numbers are conserved, is the following:

$$
\mathcal{L}=\mathcal{L}_{S M}+\sum_{i} \bar{c}_{i} O_{i} \equiv \mathcal{L}_{S M}+\Delta \mathcal{L}_{S I L H}+\Delta \mathcal{L}_{F_{1}}+\Delta \mathcal{L}_{F_{2}}
$$

with

$$
\begin{aligned}
\Delta \mathcal{L}_{S I L H}= & \frac{\bar{c}_{H}}{2 v^{2}} \partial^{\mu}\left(H^{\dagger} H\right) \partial_{\mu}\left(H^{\dagger} H\right)+\frac{\bar{c}_{T}}{2 v^{2}}\left(H^{\dagger} \overleftrightarrow{D}^{\mu} H\right)\left(H^{\dagger} \overleftrightarrow{D}_{\mu} H\right)-\frac{\bar{c}_{6} \lambda}{v^{2}}\left(H^{\dagger} H\right)^{3} \\
& +\left(\left(\frac{\bar{c}_{u}}{v^{2}} y_{u} H^{\dagger} H \bar{q}_{L} H^{c} u_{R}+\frac{\bar{c}_{d}}{v^{2}} y_{d} H^{\dagger} H \bar{q}_{L} H d_{R}+\frac{\bar{c}_{l}}{v^{2}} y_{l} H^{\dagger} H \bar{L}_{L} H l_{R}\right)+h . c .\right) \\
& +\frac{i \bar{c}_{W} g}{2 m_{W}^{2}}\left(H^{\dagger} \sigma^{i} \overleftrightarrow{D}^{\mu} H\right)\left(D^{\nu} W_{\mu \nu}\right)^{i}+\frac{i \bar{c}_{B} g^{\prime}}{2 m_{W}^{2}}\left(H^{\dagger} \overleftrightarrow{D}^{\mu} H\right)\left(\partial^{\nu} B_{\mu \nu}\right) \\
& +\frac{i \bar{c}_{H W} g}{m_{W}^{2}}\left(D^{\mu} H\right)^{\dagger} \sigma^{i}\left(D^{\nu} H\right) W_{\mu \nu}^{i}+\frac{i \bar{c}_{H B} g^{\prime}}{m_{W}^{2}}\left(D^{\mu} H\right)^{\dagger}\left(D^{\nu} H\right) B_{\mu \nu} \\
& +\frac{\bar{c}_{\gamma} g^{\prime 2}}{m_{W}^{2}} H^{\dagger} H B_{\mu \nu} B^{\mu \nu}+\frac{\bar{c}_{g} g_{S}^{2}}{m_{W}^{2}} H^{\dagger} H G_{\mu \nu}^{a} G^{a \mu \nu} \\
\Delta \mathcal{L}_{F_{1}}= & \frac{i \bar{c}_{H q}}{v^{2}}\left(\bar{q}_{L} \gamma^{\mu} q_{L}\right)\left(H^{\dagger} \overleftrightarrow{D}_{\mu} H\right)+\frac{i \bar{c}_{H q}^{\prime}}{v^{2}}\left(\bar{q}_{L} \gamma^{\mu} \sigma^{i} q_{L}\right)\left(H^{\dagger} \sigma^{i} \overleftrightarrow{D}_{\mu} H\right) \\
& +\frac{i \bar{c}_{H u}}{v^{2}}\left(\bar{u}_{R} \gamma^{\mu} u_{R}\right)\left(H^{\dagger} \overleftrightarrow{D}_{\mu} H\right)+\frac{i \bar{c}_{H d}}{v^{2}}\left(\bar{d}_{R} \gamma^{\mu} d_{R}\right)\left(H^{\dagger} \overleftrightarrow{D}_{\mu} H\right) \\
& +\left(\frac{i \bar{c}_{H u d}}{v^{2}}\left(\bar{u}_{R} \gamma^{\mu} d_{R}\right)\left(H^{c \dagger} \overleftrightarrow{D}_{\mu} H\right)+h . c .\right) \\
& +\frac{i \bar{c}_{H L}}{v^{2}}\left(\bar{L}_{L} \gamma^{\mu} L_{L}\right)\left(H^{\dagger} \overleftrightarrow{D}_{\mu} H\right)+\frac{i \bar{c}_{H L}^{\prime}}{v^{2}}\left(\bar{L}_{L} \gamma^{\mu} \sigma^{i} L_{L}\right)\left(H^{\dagger} \sigma^{i} \overleftrightarrow{D}_{\mu} H\right) \\
& +\frac{i \bar{c}_{H l}}{v^{2}}\left(\bar{l}_{R} \gamma^{\mu} l_{R}\right)\left(H^{\dagger} \overleftrightarrow{D}_{\mu} H\right),
\end{aligned}
$$

\footnotetext{
${ }^{1}$ eHDECAY is available at the following URL: http://www-itp.particle.uni-karlsruhe.de/ ${ }^{\sim}$ maggie/ eHDECAY/.
} 


$$
\begin{aligned}
\Delta \mathcal{L}_{F_{2}}= & \frac{\bar{c}_{u B} g^{\prime}}{m_{W}^{2}} y_{u} \bar{q}_{L} H^{c} \sigma^{\mu \nu} u_{R} B_{\mu \nu}+\frac{\bar{c}_{u W} g}{m_{W}^{2}} y_{u} \bar{q}_{L} \sigma^{i} H^{c} \sigma^{\mu \nu} u_{R} W_{\mu \nu}^{i}+\frac{\bar{c}_{u G} g_{S}}{m_{W}^{2}} y_{u} \bar{q}_{L} H^{c} \sigma^{\mu \nu} \lambda^{a} u_{R} G_{\mu \nu}^{a} \\
& +\frac{\bar{c}_{d B} g^{\prime}}{m_{W}^{2}} y_{d} \bar{q}_{L} H \sigma^{\mu \nu} d_{R} B_{\mu \nu}+\frac{\bar{c}_{d W} g}{m_{W}^{2}} y_{d} \bar{q}_{L} \sigma^{i} H \sigma^{\mu \nu} d_{R} W_{\mu \nu}^{i}+\frac{\bar{c}_{d G} g_{S}}{m_{W}^{2}} y_{d} \bar{q}_{L} H \sigma^{\mu \nu} \lambda^{a} d_{R} G_{\mu \nu}^{a} \\
& +\frac{\bar{c}_{l B} g^{\prime}}{m_{W}^{2}} y_{l} \bar{L}_{L} H \sigma^{\mu \nu} l_{R} B_{\mu \nu}+\frac{\bar{c}_{l W} g}{m_{W}^{2}} y_{l} \bar{L}_{L} \sigma^{i} H \sigma^{\mu \nu} l_{R} W_{\mu \nu}^{i}+\text { h.c. }
\end{aligned}
$$

The SM Lagrangian $\mathcal{L}_{S M}$ and our convention for the covariant derivatives and the gauge field strengths are reported for completeness in appendix A. In particular, $\lambda$ is the Higgs quartic coupling and the weak scale at tree level is defined to be

$$
v \equiv \frac{1}{\left(\sqrt{2} G_{F}\right)^{1 / 2}}=246 \mathrm{GeV} .
$$

By $i H^{\dagger} \overleftrightarrow{D^{\mu}} H$ we denote the Hermitian derivative $i H^{\dagger}\left(D^{\mu} H\right)-i\left(D^{\mu} H\right)^{\dagger} H$, and $\sigma^{\mu \nu} \equiv$ $i\left[\gamma^{\mu}, \gamma^{\nu}\right] / 2$. The Yukawa couplings $y_{u, d, l}$ and the Wilson coefficients $\bar{c}_{i}$ in eq. (2.3) are matrices in flavor space, and a sum over flavors has been left understood. Note that the assumption of a CP-even Higgs implies that the coefficients $\bar{c}_{u}, \bar{c}_{d}$ and $\bar{c}_{l}$ are real. As specified in eq. (2.1), we will denote as $O_{i}$ the dimension-6 operator whose coefficient is proportional to $\bar{c}_{i}$.

Our higher-dimensional Lagrangian, which is supposed to capture the leading New Physics effects, counts $12\left(\Delta \mathcal{L}_{S I L H}\right)+8\left(\Delta \mathcal{L}_{F_{1}}\right)+8\left(\Delta \mathcal{L}_{F_{2}}\right)=28$ operators. Five extra bosonic operators,

$$
\begin{array}{lll}
\frac{\bar{c}_{3 W} g^{3}}{m_{W}^{2}} \epsilon^{i j k} W_{\mu}^{i \nu} W_{\nu}^{j \rho} W_{\rho}^{k \mu}, & \frac{\bar{c}_{3 G} g_{S}^{3}}{m_{W}^{2}} f^{a b c} G_{\mu}^{a \nu} G_{\nu}^{b \rho} G_{\rho}^{c \mu}, \\
\frac{\bar{c}_{2 W}}{m_{W}^{2}}\left(D^{\mu} W_{\mu \nu}\right)^{i}\left(D_{\rho} W^{\rho \nu}\right)^{i}, & \frac{\bar{c}_{2 B}}{m_{W}^{2}}\left(\partial^{\mu} B_{\mu \nu}\right)\left(\partial_{\rho} B^{\rho \nu}\right), & \frac{\bar{c}_{2 G}}{m_{W}^{2}}\left(D^{\mu} G_{\mu \nu}\right)^{a}\left(D_{\rho} G^{\rho \nu}\right)^{a},
\end{array}
$$

which affect the gauge-boson propagators and self-interactions but with no effect on Higgs physics, should also be added to complete the operator basis, as well as 22 four-Fermi baryon-number-conserving operators. ${ }^{2}$ A comparison with ref. [26] shows that two of our operators are actually redundant. As we shall explain in more detail in section 3 (see eqs. (3.5), (3.6)), it is well known [25, 28] that two particular linear combinations of the fermionic operators in $\Delta \mathcal{L}_{F_{1}}$ are equivalent to pure oblique corrections parametrized by the operators $O_{T}, O_{W}$ and $O_{B}$ :

$$
O_{H \Psi}^{Y} \equiv \sum_{\psi} Y_{\psi} O_{H \psi} \sim O_{T}, O_{B} \quad \text { and } \quad O_{H q}^{\prime}+O_{H L}^{\prime} \sim O_{W}
$$

where the sum runs over all fermion representations, $\psi=q_{L}, u_{R}, d_{R}, L_{L}, l_{R}$, whose hypercharge has been denoted as $Y_{\psi}$. These two linear combinations have then to be excluded

\footnotetext{
${ }^{2}$ Notice that the last three operators in eq. (2.6) can be rewritten in favor of three additional independent four-Fermi operators, as in the basis of ref. [26]. The coefficients $\bar{c}_{2 W}, \bar{c}_{2 B}$ contribute respectively to the $W$ and $Y$ parameters defined in ref. [27].
} 
from $\Delta \mathcal{L}_{F_{1}}$, and we end up with exactly 53 linearly-independent operators as in ref. [26]. ${ }^{3}$ Any other dimension-six operator can be obtained from these 53 operators by using the equations of motion, or equivalently by performing appropriate field redefinitions. ${ }^{4}$

Even though our basis (2.2)-(2.4) is equivalent to the one proposed in ref. [26], we advocate that it is more appropriate for Higgs physics for at least three reasons [4]: i) Generic models of New Physics generate a contribution to the oblique $\hat{S}$ parameter [27, 29] at treelevel, which in the basis of ref. [26] would have to be encoded in the two fermionic operators $O_{H \psi}^{Y}$ and $O_{H q}^{\prime}+O_{H L}^{\prime}$ even in the absence of direct couplings between the SM fermions and the New Physics sector. There is an advantage in describing the oblique corrections in terms of the operators in (2.2) rather than in terms of the operators with fermionic currents, which generate vertex corrections and modify the Fermi constant. ii) The basis (2.1) isolates the contributions to the decays $h \rightarrow \gamma \gamma$ (from $O_{\gamma}$ ) and $h \rightarrow \gamma Z$ (from $O_{\gamma}$ and $O_{H W}-O_{H B}$ ) that occur only at the radiative level in minimally coupled theories. iii) Our basis of operators is more appropriate to establish the nature of the Higgs boson and determine the strength of its interactions. For example, as we shall explain momentarily, if the Higgs boson is a pseudo Nambu-Goldstone boson (pNGB) the coefficient of the operator $O_{\gamma}$, hence the rate $h \rightarrow \gamma \gamma$, is suppressed, while in the basis of ref. [26] this reflects into a cancellation in the linear combination $4 \bar{c}_{\gamma}+\left(\bar{c}_{W W}-\bar{c}_{W B}\right)$ (cf. footnote 4 ).

While a complete classification of the operators is essential, having a power counting to estimate their impact on physical observables, hence their relative importance, is equally crucial. In this sense a simple yet consequential observation was made in ref. [4]: when expanding the effective Lagrangian in the number of fields and derivatives, any additional power of $H$ is suppressed by a factor $g_{*} / M \equiv 1 / f$, where $g_{*} \leq 4 \pi$ denotes the coupling strength of the Higgs boson to New Physics states and $M$ is their overall mass scale; any additional derivative instead costs a factor $1 / M$. If the light Higgs boson is a composite state of the dynamics at the scale $M$, it is natural to expect $g_{*} \gg 1$, hence $f \ll M$, which implies that operators with extra powers of $H$ give the leading corrections to lowenergy observables. On the other hand, in weakly-coupled completions of the Standard Model where $g_{*} \sim g$, all operators with the same dimension can be equally important. A proper analysis of the experimental results through the language of the effective Lagrangian can thus give indication on whether the dynamics at the origin of electroweak symmetry breaking is weakly or strongly interacting. According to the power counting of ref. [4], one

\footnotetext{
${ }^{3}$ For completeness we collect in appendix $\mathrm{C}$ also the extra 6 bosonic operators of dimension-six that are CP-odd.

${ }^{4}$ In particular, the following identities hold:

$$
\begin{aligned}
& \frac{g^{2}}{4 m_{W}^{2}} H^{\dagger} H W_{\mu \nu}^{i} W^{i \mu \nu} \equiv O_{W W}=O_{W}-O_{B}+O_{H B}-O_{H W}+\frac{1}{4} O_{\gamma} \\
& \frac{g g^{\prime}}{4 m_{W}^{2}} H^{\dagger} \sigma^{i} H W_{\mu \nu}^{i} B^{\mu \nu} \equiv O_{W B}=O_{B}-O_{H B}-\frac{1}{4} O_{\gamma} .
\end{aligned}
$$


naively estimates $(\psi=u, d, l, q, L)^{5}$

$$
\begin{array}{ll}
\bar{c}_{H}, \bar{c}_{T}, \bar{c}_{6}, \bar{c}_{\psi} \sim O\left(\frac{v^{2}}{f^{2}}\right), \quad \bar{c}_{W}, \bar{c}_{B} \sim O\left(\frac{m_{W}^{2}}{M^{2}}\right), \quad \bar{c}_{H W}, \bar{c}_{H B}, \bar{c}_{\gamma}, \bar{c}_{g} \sim O\left(\frac{m_{W}^{2}}{16 \pi^{2} f^{2}}\right) \\
\bar{c}_{H \psi}, \bar{c}_{H \psi}^{\prime} \sim O\left(\frac{\lambda_{\psi}^{2}}{g_{*}^{2}} \frac{v^{2}}{f^{2}}\right), \quad \bar{c}_{H u d} \sim O\left(\frac{\lambda_{u} \lambda_{d}}{g_{*}^{2}} \frac{v^{2}}{f^{2}}\right), \quad \bar{c}_{\psi W}, \bar{c}_{\psi B}, \bar{c}_{\psi G} \sim O\left(\frac{m_{W}^{2}}{16 \pi^{2} f^{2}}\right),
\end{array}
$$

where $\lambda_{\psi}$ denotes the coupling of a generic SM fermion $\psi$ to the new dynamics. It should be stressed that these estimates are valid at the UV scale $M$, at which the effective Lagrangian is matched onto explicit models. Renormalization effects between $M$ and the EW scale mix operators with the same quantum numbers, and give in general subdominant corrections to the coefficients. We shall comment on these renormalization effects in section 4 . Notice that the estimates of $\bar{c}_{W, B}, \bar{c}_{H \psi}, \bar{c}_{H \psi}^{\prime}$ and $\bar{c}_{T}$ apply when these coefficients are generated at tree-level. However, specific symmetry protections which might be at work in the UV theory, like for example $R$-parity in SUSY theories, can force the leading corrections to arise at the 1-loop level.

Equation (2.9) suggests that in the case of a strongly-interacting light Higgs boson (SILH) the leading New Physics effects in Higgs observables are parametrized by the operators $O_{H, T, 6, \psi}$, and, if the SM fermions couple strongly to the new dynamics, by the fermionic operators of eq. (2.3) [4]. Notice that, compared to the naive counting, $\bar{c}_{H W, H B, g, \gamma}$ are suppressed by an additional factor $\left(g_{*}^{2} / 16 \pi^{2}\right)$. This is because the corresponding operators contribute to the coupling of on-shell photons and gluons to neutral particles and modify the gyromagnetic ratio of the $W$, and are thus generated only at the loop level in a minimally coupled theory. Similarly, the dipole operators of eq. (2.4) are generated at the loop-level only, hence their estimates have an extra loop factor.

A special and phenomenologically motivated case is represented by theories where the Higgs doublet is a composite Nambu-Goldstone (NG) boson of a spontaneously-broken symmetry $\mathcal{G} \rightarrow \mathcal{H}$ of the strong dynamics [4-13]. For these models the scale $f$ must be identified with the decay constant associated with the spontaneous breaking, and the naive estimate of the Wilson coefficients $\bar{c}_{i}$ is modified by the request of invariance under $\mathcal{G}$ in the limit of vanishing explicit breaking. At the level of dimension-6 operators, $O_{\gamma}, O_{g}$, $O_{6}, O_{u, d, l}$ and the dipole operators of eq. (2.4) violate the shift symmetry $H^{i} \rightarrow H^{i}+\zeta^{i}$ $\left(\zeta^{i}=\right.$ const. $)$ that is included as part of the $\mathcal{G} / \mathcal{H}$ transformations. This means that they cannot be generated in absence of an explicit breaking of the global symmetry. It follows, in particular, that the naive estimates of the operators $O_{\gamma}$ and $O_{g}$ carry in this case an additional suppression factor [4],

$$
\bar{c}_{\gamma}, \bar{c}_{g} \sim O\left(\frac{m_{W}^{2}}{16 \pi^{2} f^{2}}\right) \times \frac{g_{G_{t}}^{2}}{g_{*}^{2}},
$$

\footnotetext{
${ }^{5}$ Notice that our normalization differs from the one of ref. [4], and it is more convenient than the latter for a model-independent implementation of eq. (2.2) in a computer program. The factor multiplying each operator in the effective Lagrangian has been conveniently defined such that the dependence on $M$ and $g_{*}$ is fully encoded in the dimensionless coefficients $\bar{c}_{i}$.
} 
where $g_{G}$ denotes any weak coupling that breaks the Goldstone symmetry (one of the SM weak couplings in minimal models, i.e. the SM gauge couplings or the Yukawa couplings). The operators $O_{6}, O_{\psi}, O_{\psi G}, O_{\psi W}, O_{\psi B}$ have been defined so that their prefactor already includes one spurion coupling, precisely the Higgs quartic coupling $\lambda$ in $O_{6}$, and the Yukawa coupling $y_{\psi}$ in the other operators - indeed, both these couplings vanish for an exact NG boson. The estimates of the corresponding coefficients $\bar{c}_{6}, \bar{c}_{\psi}, \bar{c}_{\psi G}, \bar{c}_{\psi W}, \bar{c}_{\psi B}$ are thus not modified.

In writing eq. (2.2) we have assumed that each of the operators $O_{u, d, l}$ is flavor-aligned with the corresponding fermion mass term, as required in order to avoid large FlavorChanging Neutral Currents (FCNC) mediated by the tree-level exchange of the Higgs boson (see for example ref. [30] for a natural way to obtain this alignment). This implies one coefficient for the up-type quarks $\left(\bar{c}_{u}\right)$, one for down-type quarks $\left(\bar{c}_{d}\right)$, and one for the charged leptons $\left(\bar{c}_{l}\right)$, i.e. the $\bar{c}_{u, d, l}$ are proportional to the identity matrix in flavor space.

\subsection{Current bounds on flavor-preserving operators}

It is useful to review some of the most important constraints on the coefficients $\bar{c}_{i}$ that follow from current experimental results, such as electroweak precision tests, flavor data and lowenergy precision measurements. For simplicity, we focus on the bounds on flavor-conserving operators, keeping in mind that they can come also from flavor-changing processes. For a discussion of the bounds on flavor-violating operators see for example the recent review of ref. [31] as well as ref. [32].

Among the strongest bounds are those on operators that modify the vector-boson self-energies. The operator $O_{T}$, for example, violates the custodial symmetry [33] and contributes to the EW parameter $\epsilon_{1}[34,35]$. From the EW fit performed in ref. [36], it follows, with $95 \%$ probability,

$$
\Delta \epsilon_{1} \equiv \Delta \rho=\bar{c}_{T}\left(m_{Z}\right), \quad-1.5 \times 10^{-3}<\bar{c}_{T}\left(m_{Z}\right)<2.2 \times 10^{-3} .
$$

Such a stringent bound can be more naturally satisfied by assuming that the dynamics at the scale $M$ possesses an (at least approximate) $\mathrm{SU}(2)_{V}$ custodial invariance. In this case $c_{T}(M)=0$, and a non-vanishing value will be generated through the renormalization-group (RG) flow of this Wilson coefficient down to $m_{Z}$ in the presence of an explicit breaking of the custodial symmetry, as due for example to the Yukawa or hypercharge couplings. We will discuss these renormalization effects in more detail in section 4 . Notice that all the other dimension-6 operators in the effective Lagrangian are (formally) custodially symmetric and their coefficients will not be suppressed at the scale $M .{ }^{6}$ The electroweak precision tests also imply a strong bound on $O_{W}+O_{B}$ [4], since this linear combination contributes to the parameter $\epsilon_{3}[34,35]$. With $95 \%$ probability, one has [36]:

$$
\Delta \epsilon_{3}=\bar{c}_{W}\left(m_{Z}\right)+\bar{c}_{B}\left(m_{Z}\right), \quad-1.4 \times 10^{-3}<\bar{c}_{W}\left(m_{Z}\right)+\bar{c}_{B}\left(m_{Z}\right)<1.9 \times 10^{-3} .
$$

\footnotetext{
${ }^{6}$ More precisely, for all the other operators the only violation of the custodial symmetry comes from the explicit breaking due to the gauging of hypercharge. As such, this breaking is external to the EWSB dynamics, since it comes from the weak gauging of its global symmetries. Formal invariance of the operators can be restored by uplifting the hypercharge gauge field to a whole triplet of $\mathrm{SU}(2)_{R}$. The top Yukawa coupling is another source of explicit custodial breaking.
} 
From the tree-level estimate of $\bar{c}_{W, B}$ reported in eq. (2.9), and assuming an approximate custodial invariance to suppress $\bar{c}_{T}$ as explained above, it follows that eqs. (2.11) and (2.12) set a lower bound $M \gtrsim$ a few $\mathrm{TeV}$. This bound is quite robust and can be avoided only in weakly-coupled UV completions where an extra symmetry protection suppresses the leading contribution to $\bar{c}_{W, B}$ by an additional loop factor. Notable examples are SUSY theories with $R$-parity.

The fermionic operators in eq. (2.3) are strongly constrained by $Z$-pole measurements, as they modify the couplings of the $Z$ to quarks and leptons:

$$
\frac{\delta g_{L \psi}}{g_{L \psi}}=\frac{1}{2} \frac{\left(-\bar{c}_{H \Psi}+2 T_{3 L} \bar{c}_{H \Psi}^{\prime}\right)}{T_{3 L}-Q \sin ^{2} \theta_{W}}, \quad \frac{\delta g_{R \psi}}{g_{R \psi}}=\frac{1}{2} \frac{\bar{c}_{H \psi}}{Q \sin ^{2} \theta_{W}},
$$

where $T_{3 L}$ and $Q$ are respectively the $\mathrm{SU}(2)_{L}$ and electric charges of the fermion $\psi$, and $\Psi=\{L, q\}$ is the $\mathrm{SU}(2)_{L}$ doublet to which $\psi_{L}$ belongs. We used the results of ref. [36] to perform a fit on the coefficients $\bar{c}_{H \psi}, \bar{c}_{H \Psi}, \bar{c}_{H \Psi}^{\prime}$. The details of our analysis can be found in appendix D (see also ref. [37]). In the case of light quarks $(u, d, s)$ we find the following bounds

$$
\begin{array}{ll}
-0.02<\bar{c}_{H q 1}<0.03, & -0.002<\bar{c}_{H q 1}^{\prime}<0.003, \\
-0.003<\bar{c}_{H q 2}<0.006, & -0.003<\bar{c}_{H q 2}^{\prime}<0.006, \\
-0.008<\bar{c}_{H u}<0.02, & -0.03<\bar{c}_{H d}<0.02, \quad-0.03<\bar{c}_{H s}<0.02,
\end{array}
$$

while a fit on leptons and heavy quarks $(c, b)$ gives

$$
\begin{array}{ll}
-0.0003<\bar{c}_{H L}+\bar{c}_{H L}^{\prime}<0.002, & -0.002<\bar{c}_{H L}-\bar{c}_{H L}^{\prime}<0.004, \quad-0.0009<\bar{c}_{H l}<0.001, \\
-0.003<\bar{c}_{H q_{2}}-\bar{c}_{H q_{2}}^{\prime}<0.01, & -0.01<\bar{c}_{H c}<0.02, \\
-0.008<\bar{c}_{H q_{3}}+\bar{c}_{H q_{3}}^{\prime}<0.002, & -0.06<\bar{c}_{H b}<-0.009 .
\end{array}
$$

All the above bounds have $95 \%$ probability and by the various coefficients we mean their values at the scale $m_{Z}$. The weakest constraint is that on the operator $O_{H b}$, which modifies the coupling of $b_{R}$ to the $Z$ boson. The operator involving two right-handed top quarks, $O_{H t}$, is unconstrained by EW data, but it is also not relevant for the Higgs decays and will be neglected in the following. The coefficient $\bar{c}_{H t b}$ is severely constrained by the $b \rightarrow s \gamma$ rate. Indeed, the expansion of $O_{H t b}$ around the vacuum contains a vertex of the type $W t_{R} b_{R}$, which at 1-loop gives a chirally-enhanced contribution to the rate (see for example ref. [38]). We find, with $95 \%$ probability:

$$
-0.4 \times 10^{-3}<\bar{c}_{H t b}\left(m_{W}\right)<1.3 \times 10^{-3} .
$$

For a given $(v / f)$, the above bounds set a limit on the couplings of the SM fermions to the new dynamics, see eq. (2.9). Unless the scale of New Physics is very large, or some specific symmetry protection is at work in the UV theory (see for example the discussion in ref. [37]), it follows that the SM fermions must be very weakly coupled to the new dynamics, with the exception of the top quark.

The constraints on the dipole operators of eq. (2.4) come from the current experimental limits on electric dipole moments (EDMs) and anomalous magnetic moments. The bounds 
on the neutron and mercury EDMs for example strongly constrain the dipole operators with $u$ and $d$ quarks. By using the formulas of ref. [39] we find, with $95 \%$ probability, that:

$$
\begin{aligned}
& -7.01 \times 10^{-6}<\operatorname{Im}\left(\bar{c}_{u B}+\bar{c}_{u W}\right)<7.86 \times 10^{-6}, \\
& -9.42 \times 10^{-7}<\operatorname{Im}\left(\bar{c}_{d B}-\bar{c}_{d W}\right)<8.40 \times 10^{-7}, \\
& -1.62 \times 10^{-6}<\operatorname{Im}\left(\bar{c}_{u G}\right)<2.01 \times 10^{-6}, \\
& -7.71 \times 10^{-7}<\operatorname{Im}\left(\bar{c}_{d G}\right)<5.70 \times 10^{-7},
\end{aligned}
$$

where the coefficients are evaluated at the low-energy scale $\mu \sim 1 \mathrm{GeV}$. According to the naive estimate $(2.9)$, for $O(1) \mathrm{CP}$-violating phases these results imply a bound on $(v / f)^{2}$ at the level of $10^{-3}$. In natural extensions of the SM, such a strong limit clearly points to the need of a symmetry protection mechanism. For a discussion, see for example ref. [37] for the case of composite Higgs theories, and ref. [40] for the case of SUSY theories.

Among the heavier quarks the most interesting bounds are those on dipole operators with top quarks [41]. These come from the experimental limit on the neutron EDM,

$$
-1.39 \times 10^{-4}<\operatorname{Im}\left(\bar{c}_{t G}\right)<1.21 \times 10^{-4},
$$

the $b \rightarrow s \gamma$ and $b \rightarrow s l^{+} l^{-}$rates,

$$
-0.057<\operatorname{Re}\left(\bar{c}_{t W}+\bar{c}_{t B}\right)-2.65 \operatorname{Im}\left(\bar{c}_{t W}+\bar{c}_{t B}\right)<0.20,
$$

and the $t \bar{t}$ cross sections measured at the Tevatron and LHC,

$$
-6.12 \times 10^{-3}<\operatorname{Re}\left(\bar{c}_{t G}\right)<1.94 \times 10^{-3} .
$$

All these bounds have $95 \%$ probability and have been derived by making use of the formulas reported in ref. [41]. ${ }^{7}$ It is worth noting that the bounds of eqs. (2.19) and (2.20) are still about one order of magnitude weaker than the size of $\bar{c}_{t G}, \bar{c}_{t W}$ and $\bar{c}_{t B}$ expected from the naive estimate $(2.9)$ with $(v / f)^{2} \sim 0.1$. Additional weaker constraints arise from the limits on anomalous top interactions based on top decays and single top production. From the results of ref. [42] we find that, with $95 \%$ probability:

$$
-1.2<\operatorname{Re}\left(\bar{c}_{b W}\right)<1.1, \quad-0.01<\operatorname{Re}\left(\bar{c}_{t W}\right)<0.02 .
$$

where the coefficients are evaluated at the scale $\mu \sim m_{t}$.

In the lepton sector, the current measurements and SM predictions of the muon $[43,44]$ and electron [45, 46] anomalous magnetic moments and the limits on their EDMs [47-49] imply the following $95 \%$ probability bounds:

$$
\begin{aligned}
& -1.64 \times 10^{-2}<\operatorname{Re}\left(\bar{c}_{e B}-\bar{c}_{e W}\right)<3.37 \times 10^{-3}, \\
& 1.88 \times 10^{-4}<\operatorname{Re}\left(\bar{c}_{\mu B}-\bar{c}_{\mu W}\right)<6.43 \times 10^{-4}, \\
& -2.97 \times 10^{-7}<\operatorname{Im}\left(\bar{c}_{e B}-\bar{c}_{e W}\right)<4.51 \times 10^{-7}, \\
& -0.26<\operatorname{Im}\left(\bar{c}_{\mu B}-\bar{c}_{\mu W}\right)<0.29,
\end{aligned}
$$

\footnotetext{
${ }^{7}$ The coefficients are evaluated at the following scales: $\mu=m_{t}$ (eqs. (2.18) and (2.20)), $\mu=m_{W}$ (eq. (2.19)).
} 
where the coefficients are evaluated at the relevant low-energy scale. Notice that the nonvanishing value of $\operatorname{Re}\left(\bar{c}_{\mu B}-\bar{c}_{\mu W}\right)$ follows from the known $\sim 3.5 \sigma$ anomaly in the $(g-2)$ of the muon (see ref. [43] for an updated review). Among the bounds of eqs. (2.21), (2.22), (2.23) only those on $\operatorname{Im}\left(\bar{c}_{e B}-\bar{c}_{e W}\right)$ and $\operatorname{Re}\left(\bar{c}_{\mu B}-\bar{c}_{\mu W}\right)$ have the sensitivity to probe the values naively expected for these coefficients as reported in eq. (2.9). In particular, the first one sets an upper bound on $(v / f)^{2}$ of order $10^{-3}$ for an $O(1) \mathrm{CP}$ phase.

\section{Estimates of the effects on physics observables}

While the Lagrangian $\Delta \mathcal{L}=\Delta \mathcal{L}_{S I L H}+\Delta \mathcal{L}_{F_{1}}+\Delta \mathcal{L}_{F_{2}}$ is completely general, the basis of operators of eqs. (2.2)-(2.4) is particularly useful to characterize the interactions of the Higgs sector. In fact, as already anticipated, one of the main results of ref. [4] is that of identifying which operators, hence which observables, are sensitive to the strength of the Higgs interactions, rather than merely to the value of the New Physics scale $M$. In what follows we will discuss this point in greater detail and, starting from the analysis of refs. $[4,50]$, we will try to highlight a possible strategy to determine whether the dynamics behind the electroweak breaking is weak or strong. Our analysis will be based on the naive estimates of the Wilson coefficients at the matching scale. In the next section, we will discuss how the running from the matching scale to the weak scale affects these estimates.

\subsection{Operators sensitive to a strongly-interacting Higgs boson}

Let us start by considering the effects of the operators $O_{H}, O_{T}, O_{u, d, l}$ and $O_{6}$ : they modify the tree-level couplings of the Higgs boson to fermions, vector bosons and to itself. In the unitary gauge and upon canonical normalization of the Higgs kinetic term, the Lagrangian reads [51]

$$
\begin{aligned}
\mathcal{L}= & \frac{1}{2} \partial_{\mu} h \partial^{\mu} h-\frac{1}{2} m_{h}^{2} h^{2}-c_{3} \frac{1}{6}\left(\frac{3 m_{h}^{2}}{v}\right) h^{3}+\ldots \\
& +m_{W}^{2} W_{\mu}^{+} W^{-\mu}\left(1+2 c_{W} \frac{h}{v}+\ldots\right)+\frac{1}{2} m_{Z}^{2} Z_{\mu} Z^{\mu}\left(1+2 c_{Z} \frac{h}{v}+\ldots\right) \\
& -\sum_{\psi=u, d, l} m_{\psi^{(i)}} \bar{\psi}^{(i)} \psi^{(i)}\left(1+c_{\psi} \frac{h}{v}+\ldots\right)+\ldots
\end{aligned}
$$

where the Higgs couplings $c_{i=W, Z, \psi, 3}$, have been defined such that $c_{i}=1$ in the SM, and $v$ is defined by eq. (2.5). Their expressions as functions of the coefficients of the effective Lagrangian (2.2) are given in table 1. The shifts from the SM value are of order

$$
\delta c_{i} \sim \frac{g_{*}^{2} v^{2}}{M^{2}}=\frac{v^{2}}{f^{2}} .
$$

Hence, measuring the Higgs couplings probes the strength of its interactions to the new dynamics. Notice that the effective description given by $\Delta \mathcal{L}$ neglects higher powers of $(H / f)$, and is thus valid only if the shifts in the Higgs couplings are small: $\delta c_{i} \sim(v / f)^{2} \ll 1$. If the Higgs doublet is the NG boson of a spontaneously broken symmetry $\mathcal{G} \rightarrow \mathcal{H}$, on the other hand, it is possible to resum all powers of $(H / f)$ by making use of the invariance 


\begin{tabular}{|c|c|c|c|}
\hline Higgs couplings & $\Delta \mathcal{L}_{S I L H}$ & MCHM4 & MCHM5 \\
\hline$c_{W}$ & $1-\bar{c}_{H} / 2$ & $\sqrt{1-\xi}$ & $\sqrt{1-\xi}$ \\
\hline$c_{Z}$ & $1-\bar{c}_{H} / 2-2 \bar{c}_{T}$ & $\sqrt{1-\xi}$ & $\sqrt{1-\xi}$ \\
\hline$c_{\psi}(\psi=u, d, l)$ & $1-\left(\bar{c}_{H} / 2+\bar{c}_{\psi}\right)$ & $\sqrt{1-\xi}$ & $\frac{1-2 \xi}{\sqrt{1-\xi}}$ \\
\hline$c_{3}$ & $1+\bar{c}_{6}-3 \bar{c}_{H} / 2$ & $\sqrt{1-\xi}$ & $\frac{1-2 \xi}{\sqrt{1-\xi}}$ \\
\hline$c_{g g}$ & $8\left(\alpha_{s} / \alpha_{2}\right) \bar{c}_{g}$ & 0 & 0 \\
\hline$c_{\gamma \gamma}$ & $8 \sin ^{2} \theta_{W} \bar{c}_{\gamma}$ & 0 & 0 \\
\hline$c_{Z \gamma}$ & $\left(\bar{c}_{H B}-\bar{c}_{H W}-8 \bar{c}_{\gamma} \sin ^{2} \theta_{W}\right) \tan \theta_{W}$ & 0 & 0 \\
\hline$c_{W W}$ & $-2 \bar{c}_{H W}$ & 0 & 0 \\
\hline$c_{Z Z}$ & $-2\left(\bar{c}_{H W}+\bar{c}_{H B} \tan ^{2} \theta_{W}-4 \bar{c}_{\gamma} \tan ^{2} \theta_{W} \sin ^{2} \theta_{W}\right)$ & 0 & 0 \\
\hline$c_{W \partial W}$ & $-2\left(\bar{c}_{W}+\bar{c}_{H W}\right)$ & 0 & 0 \\
\hline$c_{Z \partial Z}$ & $-2\left(\bar{c}_{W}+\bar{c}_{H W}\right)-2\left(\bar{c}_{B}+\bar{c}_{H B}\right) \tan ^{2} \theta_{W}$ & 0 & 0 \\
\hline$c_{Z \partial \gamma}$ & $2\left(\bar{c}_{B}+\bar{c}_{H B}-\bar{c}_{W}-\bar{c}_{H W}\right) \tan \theta_{W}$ & 0 & 0 \\
\hline
\end{tabular}

Table 1. The second column reports the values of the Higgs couplings $c_{i}$ defined in eq. (3.23) in terms of the coefficients $\bar{c}_{i}$ of the effective Lagrangian $\Delta \mathcal{L}_{S I L H}$. The last two columns show the predictions of the MCHM4 and MCHM5 models in terms of $\xi=(v / f)^{2}$; the effects of the heavy resonances have been neglected for simplicity, so that only the couplings $c_{W, Z, \psi, 3}$ are non-vanishing. The auxiliary parameter $\alpha_{2}$ is defined by eq. (3.20).

under (non-linear) $\mathcal{G}$ transformations. Such an improved effective Lagrangian thus relies only on the expansion in the number of derivatives. For example, in models based on the $\mathrm{SO}(5) / \mathrm{SO}(4)$ coset $[13,52]$ the couplings of the Higgs boson to $W$ and $Z$ are predicted to be $c_{W}=c_{Z} \equiv c_{V}=\sqrt{1-\xi}$, where $\xi \equiv(v / f)^{2}$. The couplings to fermions, on the other hand, are not uniquely fixed by the choice of the coset, but depend on how the SM fermions are coupled to the strong dynamics. The last two columns of table 1 report the predictions of the Minimal Composite Higgs Model MCHM4 [13] and MCHM5 [52], where the SM fermions couple linearly to composite operators transforming as the spinorial and fundamental representations of $\mathrm{SO}(5)$, respectively. For simplicity, the predictions are derived by including only the effects of the Higgs non-linearities, and neglecting those from 
the heavy resonances, hence only the coefficients $c_{V}, c_{\psi}$ and $c_{3}$ are non-vanishing. The models MCHM4 and MCHM5 will be considered as benchmarks in the rest of this work.

In general, a shift of the tree-level Higgs couplings of order $(v / f)^{2}$ implies that the theory gets strongly coupled at energies $\sim 4 \pi f$, unless new weakly-coupled physics states set in to regulate the energy growth of the scattering amplitudes. The dominant effect comes from the energy growth of the $V_{L} V_{L} \rightarrow V_{L} V_{L}\left(V=W^{ \pm}, Z^{0}\right)$ scattering amplitudes, which become non-perturbative at the scale $\Lambda_{s}=4 \pi v / \sqrt{\left|\bar{c}_{H}\right|}$. A modified coupling to the top quark leads instead to strong $V_{L} V_{L} \rightarrow t \bar{t}$ scattering at energies of order $\Lambda_{s}=$ $16 \pi^{2} v^{2} /\left(m_{t} \sqrt{\left|\bar{c}_{u}+\bar{c}_{H}\right|}\right)$. The scale of New Physics is thus required to lie below, or at, such ultimate range of validity of the effective theory: $M \lesssim \Lambda_{s}$.

\subsection{Operators sensitive to the scale of New Physics}

The operators $O_{W}, O_{B}$ can be generated at tree-level by the exchange of heavy particles, for example heavy spin- 1 states. In the unitary gauge they are written in terms of the following three operators ${ }^{8}$

$$
\left(D^{\mu} W_{\mu \nu}^{+}\right) W^{-\nu} h, \quad\left(\partial^{\mu} Z_{\mu \nu}\right) Z^{\nu} h, \quad\left(\partial^{\mu} \gamma_{\mu \nu}\right) Z^{\nu} h
$$

plus terms with zero or two Higgs fields. The fact that there are three possible operators in the unitary gauge indicates that their coefficients are related by one identity if the Higgs boson belongs to an $\mathrm{SU}(2)$ doublet, see eq. (3.25). We will discuss this point in greater detail in section 3.6.

It is easy to see that $O_{W}, O_{B}$ give corrections to the tree-level Higgs couplings and generate quartic interactions with one vector boson and two SM fermions that contribute to the three-body decays $h \rightarrow V V^{*} \rightarrow V \psi \bar{\psi}^{9}$ Indeed, by making use of the equations of motion, ${ }^{10}$

$$
i D^{\mu} W_{\mu \nu}^{i}=g H^{\dagger} \frac{\sigma^{i}}{2} \overleftrightarrow{D}_{\nu} H-i g \bar{\psi} \frac{\sigma^{i}}{2} \gamma_{\nu} \psi, \quad i \partial^{\mu} B_{\mu \nu}=\frac{g^{\prime}}{2} H^{\dagger} \overleftrightarrow{D}_{\nu} H-i g^{\prime} \bar{\psi} Y \gamma_{\nu} \psi
$$

one can rewrite $O_{W}$ and $O_{B}$ as

$$
\begin{aligned}
O_{W} & =-2 O_{H}+\frac{4}{v^{2}}\left(H^{\dagger} H\right)\left|D_{\mu} H\right|^{2}+O_{H q}^{\prime}+O_{H L}^{\prime} \\
O_{B} & =2 \tan ^{2} \theta_{W}\left(-O_{T}+O_{H \Psi}^{Y}\right)
\end{aligned}
$$

where the linear combination $O_{H \Psi}^{Y}$ has been defined in eq. (2.7). Upon the field redefinition $H \rightarrow H-2 \bar{c}_{W}\left(H^{\dagger} H\right) H / v^{2}$, the operator $\left(H^{\dagger} H\right)\left|D_{\mu} H\right|^{2}$ can be rewritten in terms of those

\footnotetext{
${ }^{8}$ Here and in the following, derivatives acting on operators in the unitary gauge are covariant under local $\mathrm{U}(1)_{e m}$ transformations. Operators like $\left(\partial^{\mu} Z_{\mu \nu}\right) \gamma^{\nu} h$ or $\left(\partial^{\mu} \gamma_{\mu \nu}\right) \gamma^{\nu} h$ obviously cannot be generated since they break the $\mathrm{U}(1)_{\text {em }}$ local symmetry.

${ }^{9}$ We thank Riccardo Rattazzi for pointing this out to us.

${ }^{10}$ For simplicity we have left a sum over all fermion representations $\psi$ understood in eq. (3.4).
} 
in eq. (2.2). Specifically, eq. (3.5) becomes: $:^{11}$

$$
O_{W}=-6 O_{H}+2\left(\left(O_{u}+O_{d}+O_{l}\right)+\text { h.c. }\right)-8 O_{6}+O_{H q}^{\prime}+O_{H L}^{\prime} .
$$

From the estimates of $\bar{c}_{W}, \bar{c}_{B}$ and $\bar{c}_{H}, \bar{c}_{T}, \bar{c}_{\psi}, \bar{c}_{6}$ in eq. (2.9) one can see that the shifts to the tree-level Higgs couplings due to $O_{W}, O_{B}$ are of order $\left(m_{W} / M\right)^{2}$, hence subdominant in the case of a strongly interacting Higgs boson. Notice that the couplings of the Higgs boson to $W$ and $Z$ get different shifts from $O_{B}\left(\right.$ since $\left.\Delta \bar{c}_{T} \neq 0\right)$. In practice, the constraint $(2.12)$ bounds this custodial-symmetry breaking effect down to an unobservable level, unless some fine tuning is in place in the combination $\bar{c}_{W}+\bar{c}_{B}$ so that $\bar{c}_{B}$ can be large. Notice that despite the operator $O_{T}$ is generated after using the equations of motion, its contribution to $\Delta \epsilon_{1}$ (corresponding to a non-vanishing $\hat{T}$ parameter $[27,29]$ ) is exactly canceled by the vertex correction implied by the linear combination of fermionic operators which is also generated. ${ }^{12}$ This is of course expected, since $O_{W}, O_{B}$ only contribute to $\epsilon_{3}$, and not to $\epsilon_{1}$.

In general, the contribution of $O_{W}, O_{B}$ to inclusive observables, in particular to the Higgs decay rates, is of order $\left(m_{W}^{2} / M^{2}\right)$ :

$$
\left.\frac{\delta \Gamma(h \rightarrow V V)}{\Gamma(h \rightarrow V V)}\right|_{O_{W}, O_{B}} \sim O\left(\frac{m_{W}^{2}}{M^{2}}\right)
$$

where in this case $V V=W^{(*)} W^{*}, Z^{(*)} Z^{*}, Z^{(*)} \gamma, \gamma \gamma$. This implies that these operators are sensitive only to the value of the scale of New Physics $M$, and do not probe the coupling strength $g_{*}$. From the quantitative side, the constraint (2.12) suggests that their effects in inclusive Higgs decay rates is too small to be observable. For example, we find that for small $\bar{c}_{W, B}$ the tree-level correction to the $W W$ and $Z Z$ partial rates is well approximated by: ${ }^{13}$

$$
\frac{\Gamma\left(h \rightarrow W^{(*)} W^{*}\right)}{\Gamma\left(h \rightarrow W^{(*)} W^{*}\right)_{S M}} \simeq 1+2.2 \bar{c}_{W}, \quad \frac{\Gamma\left(h \rightarrow Z^{(*)} Z^{*}\right)}{\Gamma\left(h \rightarrow Z^{(*)} Z^{*}\right)_{S M}} \simeq 1+2.0\left(\bar{c}_{W}+\tan ^{2} \theta_{W} \bar{c}_{B}\right) .
$$

Notice that despite its custodial invariance, the operator $O_{W}$ affects in a slightly different way the decay of the Higgs boson into $W W$ and $Z Z$, due to the fact that at least one of the two final vector bosons is off-shell. ${ }^{14}$ At the one-loop level $O_{W}$ also contributes to the

\footnotetext{
${ }^{11}$ By means of eqs. (3.6) and (3.8) it is thus always possible to remove $O_{W}$ and $O_{B}$ provided the coefficients of the other operators are shifted as follows: $\bar{c}_{i} \rightarrow \bar{c}_{i}+\Delta \bar{c}_{i}$, with

$$
\begin{aligned}
\Delta \bar{c}_{H} & =-6 \bar{c}_{W}, \quad \Delta \bar{c}_{T}=-2 \tan ^{2} \theta_{W} \bar{c}_{B}, \quad \Delta \bar{c}_{6}=-8 \bar{c}_{W}, \quad \Delta \bar{c}_{\psi}=2 \bar{c}_{W} \\
\Delta \bar{c}_{H q}^{\prime} & =\Delta \bar{c}_{H L}^{\prime}=\bar{c}_{W} \\
6 \Delta \bar{c}_{H q} & =\frac{3}{2} \Delta \bar{c}_{H u}=-3 \Delta \bar{c}_{H d}=-2 \Delta \bar{c}_{H L}=-\Delta \bar{c}_{H l}=-2 \tan ^{2} \theta_{W} \bar{c}_{B} .
\end{aligned}
$$

${ }^{12}$ See for example eq. (9.10) of ref. [28].

${ }^{13}$ Here and in the following our approximated formulas have been obtained by using eHDECAY [16] with $m_{h}=125 \mathrm{GeV}$. QCD corrections to the decay rates are fully included. Electroweak corrections are instead not included, since their effect on the numerical prefactor appearing in front of the coefficients $\bar{c}_{i}$ is of order $\left(v^{2} / f^{2}\right)\left(\alpha_{2} / 4 \pi\right)$ and thus beyond the accuracy of our computation. See ref. [16] for more details.

${ }^{14}$ It is easy to check that for $m_{h}>2 m_{Z}$ and on-shell decays one has:

$$
\frac{\Gamma(h \rightarrow W W)}{\Gamma(h \rightarrow W W)_{S M}} \simeq 1+4 \bar{c}_{W}, \quad \frac{\Gamma(h \rightarrow Z Z)}{\Gamma(h \rightarrow Z Z)_{S M}} \simeq 1+4\left(\bar{c}_{W}+\tan ^{2} \theta_{W} \bar{c}_{B}\right) .
$$

These formulas coincide with those of eqs. (79)-(80) of ref. [4], which are thus valid only for on-shell decays. 
Higgs decays into $Z \gamma$ and $\gamma \gamma$ (while $O_{B}$ does not). We find:

$$
\frac{\Gamma(h \rightarrow Z \gamma)}{\Gamma(h \rightarrow Z \gamma)_{S M}} \simeq 1+4.2 \bar{c}_{W}, \quad \frac{\Gamma(h \rightarrow \gamma \gamma)}{\Gamma(h \rightarrow \gamma \gamma)_{S M}} \simeq 1+5.0 \bar{c}_{W}
$$

which agree with eqs. (82) and (83) of ref. [4]. ${ }^{15}$ For $\bar{c}_{W, B} \sim 10^{-3}$ the above approximate formulas imply corrections too small to be observed at the LHC. On the other hand, one could try to take advantage of the different predictions in terms of angular and invariant mass distributions which are implied by the dimension- 6 operators compared to the treelevel SM prediction. The most promising strategy could be in fact that based on the analysis of the angular distributions of the final fermions [53-55]. In the ideal case in which one is able to kill completely the SM tree-level contribution by means of appropriate kinematic cuts, the relative effect of NP becomes of order

$$
\frac{d \Gamma(h \rightarrow V V)}{d \Omega} /\left(\frac{d \Gamma(h \rightarrow V V)}{d \Omega}\right)_{S M} \lesssim 1+\bar{c}_{W, B} \frac{16 \pi^{2}}{g^{2}}
$$

which might leave room for observable effects even for $\bar{c}_{W, B} \sim O\left(10^{-3}\right)$. Clearly, a more precise assessment of the efficiency of such a strategy requires a dedicated analysis [56].

\subsection{Operators generated at the one-loop level}

Let us now focus on the operators $O_{H W}, O_{H B}, O_{\gamma}$ and $O_{g}$, which are generated at the one-loop level. In the unitary gauge, $O_{H W, H B, \gamma}$ are rewritten in terms of

$$
W_{\mu \nu}^{+} W^{-\mu \nu} h, \quad Z_{\mu \nu} Z^{\mu \nu} h, \quad \gamma_{\mu \nu} \gamma^{\mu \nu} h, \quad Z_{\mu \nu} \gamma^{\mu \nu} h
$$

plus other terms with zero or two Higgs fields. Since the coefficients of the above four operators are functions of $\bar{c}_{H W}, \bar{c}_{H B}$ and $\bar{c}_{\gamma}$, they are related by one identity, see eq. (3.24). We will discuss this point in greater detail in section 3.6.

As implied from the naive estimates (2.9), the contribution of $O_{H W, H B, \gamma}$ to the $W W$ and $Z Z$ inclusive rates is of order $(V V=W W, Z Z)$

$$
\left.\frac{\delta \Gamma(h \rightarrow V V)}{\Gamma(h \rightarrow V V)}\right|_{O_{\gamma}, O_{H W}, O_{H B}} \sim O\left(\frac{m_{W}^{2}}{16 \pi^{2} f^{2}}\right)
$$

Although such an effect depends on the Higgs interaction strength, it is suppressed com-

\footnotetext{
${ }^{15}$ The easiest way to compute the one-loop contribution of $O_{W}$ to the $Z \gamma$ and $\gamma \gamma$ rates is by using eq. (3.5) to rewrite this operator in terms of the others. Among the operators generated in this way, only $O_{H}$ gives a contribution. Notice that if eq. (3.8) is used instead, one has to take into account also the contribution of $\left(O_{u}+O_{d}+O_{l}\right)$ and the shift to the Fermi constant induced by $O_{H q}^{\prime}+O_{H L}^{\prime}$.
} 
pared to eq. (3.9) by a loop factor. We find that the following approximate formulas hold ${ }^{16}$

$$
\begin{aligned}
\frac{\Gamma\left(h \rightarrow W^{(*)} W^{*}\right)}{\Gamma\left(h \rightarrow W^{(*)} W^{*}\right)_{S M}} & \simeq 1+3.7 \bar{c}_{H W}, \\
\frac{\Gamma\left(h \rightarrow Z^{(*)} Z^{*}\right)}{\Gamma\left(h \rightarrow Z^{(*)} Z^{*}\right)_{S M}} & \simeq 1+3.0\left(\bar{c}_{H W}+\tan ^{2} \theta_{W} \bar{c}_{H B}\right)-0.26 \bar{c}_{\gamma} .
\end{aligned}
$$

While the contribution due to $\bar{c}_{H B}$ and $\bar{c}_{\gamma}$ explicitly violates the custodial symmetry and thus differentiates $W W$ from $Z Z$, the different numerical factor multiplying $\bar{c}_{H W}$ in the two formulas above is due to the off-shellness of at least one of the two vector bosons, similarly to eq. (3.10). Although there is currently no stringent bound on the coefficients $\bar{c}_{H W, H B, \gamma}$, the estimate (2.9) suggests that their correction to inclusive rates is unobservable at the LHC. As discussed in the previous section, on the other hand, a study of the angular and invariant mass distributions of these decays can potentially uncover the effect of New Physics. In particular, an estimate similar to that of eq. (3.13) can be derived also for $\bar{c}_{H W, H B, \gamma}$.

The processes $h \rightarrow \gamma \gamma, h \rightarrow Z \gamma$ and $h \rightarrow g g$ (or equivalently $g g \rightarrow h$ ) can in principle test the Higgs interaction strengths much more powerfully, since they arise at the one-loop level in the SM. Naively one expects:

$$
\left.\frac{\delta \Gamma(h \rightarrow g g, \gamma \gamma, Z \gamma)}{\Gamma(h \rightarrow g g, \gamma \gamma, Z \gamma)}\right|_{O_{g}, O_{\gamma}, O_{H W}, O_{H B}} \sim O\left(\frac{v^{2}}{f^{2}}\right) .
$$

We find that the following approximate formulas hold to good accuracy for small $\bar{c}_{i}$ 's:

$$
\begin{aligned}
& \frac{\Gamma(h \rightarrow g g)}{\Gamma(h \rightarrow g g)_{S M}} \simeq 1+22.2 \bar{c}_{g} \frac{4 \pi}{\alpha_{2}} \\
& \frac{\Gamma(h \rightarrow \gamma \gamma)}{\Gamma(h \rightarrow \gamma \gamma)_{S M}} \simeq 1-0.54 \bar{c}_{\gamma} \frac{4 \pi}{\alpha_{e m}}, \\
& \frac{\Gamma(h \rightarrow Z \gamma)}{\Gamma(h \rightarrow Z \gamma)_{S M}} \simeq 1+0.19\left(\bar{c}_{H W}-\bar{c}_{H B}+8 \bar{c}_{\gamma} \sin ^{2} \theta_{W}\right) \frac{4 \pi}{\sqrt{\alpha_{2} \alpha_{e m}}},
\end{aligned}
$$

where we have conveniently defined

$$
\alpha_{2} \equiv \frac{\sqrt{2} G_{F} m_{W}^{2}}{\pi}
$$

and by $\alpha_{e m}$ we indicate the value of the running electromagnetic coupling $\alpha_{e m}\left(q^{2}=0\right)$ in the Thomson limit. If the Higgs boson is a NG boson, the coefficients $\bar{c}_{g}$ and $\bar{c}_{\gamma}$ are further

\footnotetext{
${ }^{16}$ For $m_{h}>2 m_{Z}$ and on-shell decays, we find instead

$$
\begin{aligned}
\frac{\Gamma(h \rightarrow W W)}{\Gamma(h \rightarrow W W)_{S M}} & \simeq 1+8 \bar{c}_{H W}, \\
\frac{\Gamma(h \rightarrow Z Z)}{\Gamma(h \rightarrow Z Z)_{S M}} & \simeq 1+8\left(\bar{c}_{H W}+\tan ^{2} \theta_{W} \bar{c}_{H B}\right)-16 \tan ^{2} \theta_{W} \sin ^{2} \theta_{W} \bar{c}_{\gamma} .
\end{aligned}
$$

Comparing with the analog formulas in eqs. (79) and (80) of ref. [4], we find that in these latter there is a missing factor 2 and the term proportional to $\bar{c}_{\gamma}$ was not included either. Notice also that the effect of the off-shellness of the gauge bosons is rather large, as one can see by comparing eq. (3.16) with eq. (3.17).
} 
suppressed by a factor $\left(g_{G_{*}} / g_{*}\right)^{2}$, see eq. (2.10), where $g_{G_{t}}$ is a weak coupling. This implies that in this class of theories the corrections to $\Gamma(h \rightarrow \gamma \gamma)$ and $\Gamma(h \rightarrow g g)$ depend only on the scale of New Physics and not on the Higgs interaction strength. In fact, in the case of minimal models with linear couplings, like for example the MCHM4 and MCHM5, the low energy theorem [57-59] implies that the leading contribution to the $\gamma \gamma$ and $g g$ decay rates from the virtual exchange of heavy fermions is additionally suppressed [60-63] due to a cancellation between the effect parametrized by $\bar{c}_{g, \gamma}$ and the one that follows from the shift in the top Yukawa coupling due to $\bar{c}_{u}$ and $\bar{c}_{H}$ (see ref. [62] for an interesting exception). In general, in theories with a pNGB Higgs boson the local corrections to the rates $\Gamma(h \rightarrow \gamma \gamma)$ and $\Gamma(h \rightarrow g g)$ from $O_{\gamma}$ and $O_{g}$ are expected to be small and subdominant compared to the effect from the modified tree-level Higgs couplings.

\subsection{Fermionic operators}

The fermionic operators in $\Delta \mathcal{L}_{F_{1}}$ are sensitive to the strength of the couplings of the Higgs boson and of the SM fermions to the new dynamics. They lead to contact corrections to the three-body decays $h \rightarrow V V^{*} \rightarrow V \psi \psi$ which are naively of order

$$
\frac{\delta \Gamma(h \rightarrow V \bar{\psi} \psi)}{\Gamma(h \rightarrow V \bar{\psi} \psi)} \sim O\left(\frac{v^{2}}{f^{2}} \frac{\lambda_{\psi}^{2}}{g_{*}^{2}}\right) .
$$

Compared to the corrections from $O_{W}$ and $O_{B}$, the effect of the fermionic operators is potentially enhanced by a factor $\left(\lambda_{\psi}^{2} / g^{2}\right)$. In practice, the possibility of large fermionic couplings $\lambda_{\psi}$ is strongly constrained by LEP, see eqs. (2.14)-(2.16). Scenarios in which a large degree of compositeness of either the left- or right-handed quarks is not ruled out are generically those in which the corresponding operators in $\Delta \mathcal{L}_{F_{1}}$ are not generated as due to some protecting symmetry (see for example refs. [37, 64, 65]). Large corrections to the inclusive rate of the three-body decays $h \rightarrow V \bar{\psi} \psi$ from $\Delta \mathcal{L}_{F_{1}}$ are thus excluded, while the possibility of detecting the effects of these operators through the analysis of differential distributions should be explored, similarly to what has been discussed for $O_{W}$ and $O_{B}$.

Among the dipole operators in $\Delta \mathcal{L}_{F_{2}}$, those with light fermions are already strongly constrained by current precision data, but potentially sizable effects could still come from the operators involving the top quark. For example, the contribution of $O_{t G}$ to $g g \rightarrow h$, $g g \rightarrow t \bar{t}, g g \rightarrow t \bar{t} h$ is of order $E^{2} /\left(16 \pi^{2} f^{2}\right)$, where $E$ is the energy scale relevant in the process. More in detail

$$
\frac{\delta \sigma(g g \rightarrow h)}{\sigma(g g \rightarrow h)} \sim \hat{c}_{t G}, \quad \frac{\delta \sigma(g g \rightarrow t \bar{t})}{\sigma(g g \rightarrow t \bar{t})} \sim \hat{c}_{t G} \frac{\sqrt{s}}{m_{t}}, \quad \frac{\delta \sigma(g g \rightarrow t \bar{t} h)}{\sigma(g g \rightarrow t \bar{t} h)} \sim \hat{c}_{t G} \frac{s}{m_{t}^{2}},
$$

where we have defined $\hat{c}_{t G} \equiv \operatorname{Re}\left(\bar{c}_{t G}\right)\left(m_{t}^{2} / m_{W}^{2}\right) \sim m_{t}^{2} /\left(16 \pi^{2} f^{2}\right) \simeq 3 \times 10^{-3}\left(v^{2} / f^{2}\right)$. Notice that the experimental limit on the neutron EDM puts an upper bound on the imaginary part of $\hat{c}_{t G}$ at the $10^{-4}$ level, see eq. (2.18), which indicates that this is currently the most sensitive experiment on $\operatorname{Im}\left(\bar{c}_{t G}\right)$. Some mechanism is however required to suppress the imaginary parts of the dipole operators involving light fermions, in order to satisfy the stringent constraints of eq. (2.17). By the same mechanism also $\operatorname{Im}\left(\bar{c}_{t G}\right)$ could be suppressed, so that the processes of eq. (3.22) are essential to probe the contribution of $O_{t G}$ 
due to $\operatorname{Re}\left(\bar{c}_{t G}\right)$. From eq. (3.22) and the naive estimate of $\hat{c}_{t G}$ it follows that the most sensitive process is perhaps $g g \rightarrow t \bar{t}$, in particular the events at large invariant mass, although a precision larger than the one currently achieved is required to constrain $(v / f)$. To this aim, the analysis of differential distributions and spin correlations could be a successful strategy $[41,66-68]$. The NP contribution to the process $g g \rightarrow t \bar{t} h$ can in principle get the largest enhancement from a cut on $\sqrt{s}$, but the small rate might limit the actual sensitivity achievable at the LHC [69]. Finally, additional information comes from the experimental limits on top anomalous couplings obtained at the Tevatron and the LHC, although their sensitivity on NP is expected to be much smaller by naive estimate. The operator $O_{t W}$, in particular, gives the largest effect and generates the anomalous coupling $g_{R}\left(g / m_{W}\right) \bar{b}_{L} \sigma^{\mu \nu} W_{\mu \nu}^{-} t_{R}$ [42]. Naively one expects $g_{R}=\left(4 m_{t} / m_{W}\right) \bar{c}_{t W} \sim m_{t} m_{W} /\left(16 \pi^{2} f^{2}\right)=1.5 \times 10^{-3}(v / f)^{2}$, an effect too small to be observed even for $f$ of order $v$.

\subsection{Non-linear Lagrangian for a Higgs-like scalar}

Summarizing, by working in the unitary gauge and in the basis of fermion mass eigenstates, the effective Lagrangian relevant for Higgs physics reads as follows [51]

$$
\begin{aligned}
\mathcal{L}= & \frac{1}{2} \partial_{\mu} h \partial^{\mu} h-\frac{1}{2} m_{h}^{2} h^{2}-c_{3} \frac{1}{6}\left(\frac{3 m_{h}^{2}}{v}\right) h^{3}-\sum_{\psi=u, d, l} m_{\psi^{(i)}} \bar{\psi}^{(i)} \psi^{(i)}\left(1+c_{\psi} \frac{h}{v}+\ldots\right) \\
& +m_{W}^{2} W_{\mu}^{+} W^{-\mu}\left(1+2 c_{W} \frac{h}{v}+\ldots\right)+\frac{1}{2} m_{Z}^{2} Z_{\mu} Z^{\mu}\left(1+2 c_{Z} \frac{h}{v}+\ldots\right)+\ldots \\
& +\left(c_{W W} W_{\mu \nu}^{+} W^{-\mu \nu}+\frac{c_{Z Z}}{2} Z_{\mu \nu} Z^{\mu \nu}+c_{Z \gamma} Z_{\mu \nu} \gamma^{\mu \nu}+\frac{c_{\gamma \gamma}}{2} \gamma_{\mu \nu} \gamma^{\mu \nu}+\frac{c_{g g}}{2} G_{\mu \nu}^{a} G^{a \mu \nu}\right) \frac{h}{v} \\
& +\left(c_{W \partial W}\left(W_{\nu}^{-} D_{\mu} W^{+\mu \nu}+h . c .\right)+c_{Z \partial Z} Z_{\nu} \partial_{\mu} Z^{\mu \nu}+c_{Z \partial \gamma} Z_{\nu} \partial_{\mu} \gamma^{\mu \nu}\right) \frac{h}{v}+\ldots
\end{aligned}
$$

where, we recall, $v$ is defined in eq. (2.5). We have shown only terms involving up to three bosonic fields, and we have omitted in particular those involving fermions that follow from $\Delta \mathcal{L}_{F_{1}}+\Delta \mathcal{L}_{F_{2}}$. Their form can be easily derived from eqs. (2.3) and (2.4). The relations between the couplings appearing in eq. (3.23) and the coefficients of the dimension-6 operators in eq. (2.2) are reported in table 1. It is worth noting that the same Lagrangian (3.23) applies also to the case in which the electroweak symmetry $\mathrm{SU}(2)_{L} \times \mathrm{U}(1)_{Y}$ is non-linearly realized and $h$ is a generic CP-even scalar, singlet of the custodial symmetry, not necessarily connected with the EW symmetry breaking. Indeed, each of the terms in (3.23), being invariant under local U(1)em transformations, can be dressed up with the Nambu-Goldstone bosons that are eaten to form the longitudinal $W$ and $Z$ polarizations and made manifestly $\mathrm{SU}(2)_{L} \times \mathrm{U}(1)_{Y}$ gauge invariant [70,71] (see also ref. [72]). The explicit expression in such a basis has been given in refs. [73, 74] at the level of four-derivative operators. In this sense the effective Lagrangian (3.23) is a generic tool to understand the origin of the newly discovered boson and the role it plays in the electroweak symmetry breaking dynamics. It is valid for arbitrary values of the couplings $c_{i}$ appearing in eq. (3.23), and it can be used to make computations of observable quantities at a given order in an expansion in $E / M$ and in $\alpha_{S M} / 4 \pi$, where by the latter we indicate the generic SM loop expansion parameter. 
That is in full analogy with other well-known effective theories, see ref. [3]. It should be stressed that, according to a well established methodology and similarly to eq. (2.2), in this effective Lagrangian all quantum fluctuations associated to short-length modes (highenergy modes) have already been considered and are parametrized by local operators with an increasing number of derivatives, while quantum fluctuations (loop diagrams) involving the light modes still have to be taken into account. For instance, top loops will give an additional contribution to the on-shell $h$-gluon-gluon coupling. While eq. (3.23) is general, the effective Lagrangian (2.2) assumes that $h$ is part of an $\mathrm{SU}(2)_{L}$ doublet and further relies on the expansion in powers of $H / f$. As such, it is valid only in the limit of small deviations of the Higgs couplings from their SM values and up to corrections of order $O\left(v^{2} / f^{2}\right)$.

\subsection{Implications of custodial symmetry}

Another difference between the non-linear Lagrangian (3.23) and the SILH Lagrangian (2.2) is that the first one contains two more free parameters. This means that there are two relations among the couplings of eq. (3.23) which hold at the level of dimension-6 operators if the Higgs is part of a doublet. As noticed in sections (3.2) and (3.3), the first identity relates $c_{W W}, c_{Z Z}, c_{Z \gamma}$ and $c_{\gamma \gamma}$, while the second relates $c_{W \partial W}, c_{Z \partial Z}$ and $c_{Z \partial \gamma}$. They read:

$$
\begin{aligned}
c_{W W}-c_{Z Z} \cos ^{2} \theta_{W} & =c_{Z \gamma} \sin 2 \theta_{W}+c_{\gamma \gamma} \sin ^{2} \theta_{W} \\
c_{W \partial W}-c_{Z \partial Z} \cos ^{2} \theta_{W} & =\frac{c_{Z \partial \gamma}}{2} \sin 2 \theta_{W} .
\end{aligned}
$$

In fact both identities are not special to the case in which the Higgs is a doublet, but are a general consequence of custodial symmetry. This latter is accidental in the SILH Lagrangian if one restricts to the operators that lead to derivative couplings of the Higgs to vector bosons. Starting at the dimension- 8 order, it is possible to write cutodial-breaking operators that lead to couplings that violate the relations (3.24) and (3.25). For instance

$$
\frac{\bar{c}_{8 W W} g^{2}}{m_{W}^{2} v^{2}}\left(H^{\dagger} W_{\mu \nu}^{a} \sigma^{a} H\right)\left(H^{\dagger} W^{b \mu \nu} \sigma^{b} H\right)+\frac{i \bar{c}_{8 W} g}{v^{2} m_{W}^{2}}\left(H^{\dagger} \sigma^{a} H\right)\left(D^{\mu} W_{\mu \nu}\right)^{a}\left(H^{\dagger} \overleftrightarrow{D^{\nu}} H\right)
$$

gives rise to

$$
\begin{array}{rlrl}
c_{Z \partial Z} & =-4 \bar{c}_{8 W}, & c_{Z \partial \gamma} & =-4 \tan \theta_{W} \bar{c}_{8 W}, \\
c_{Z Z} & =8 \cos ^{2} \theta_{W} \bar{c}_{8 W W}, \quad c_{Z \gamma}=4 \sin 2 \theta_{W} \bar{c}_{8 W W}, \quad c_{\gamma \gamma}=8 \sin ^{2} \theta_{W} \bar{c}_{8 W W},
\end{array}
$$

and the relations (3.24) and (3.25) are not fulfilled. ${ }^{17}$

A third relation holds on the non-derivative couplings $c_{W}$ and $c_{Z}$ if one assumes that custodial symmetry is an invariance of the Lagrangian $(2.2)$, so that $\bar{c}_{T}=0$; it reads:

$$
c_{W}=c_{Z}
$$

As said above, while all three identities (3.24), (3.25) and (3.28) are a consequence of custodial symmetry, the first two are accidental at the level of dimension- 6 operators if the Higgs is part of a doublet.

\footnotetext{
${ }^{17}$ The two operators in (3.26) give rise to the oblique parameter $\hat{U}$, see for instance ref. [27]: $\hat{U}=$ $-\bar{c}_{8 W}-2 \bar{c}_{8 H W}$ while $\hat{S}=\bar{c}_{8 H W}$.
} 
To show that eqs. (3.24), (3.25) and (3.28) follow from custodial invariance, let us consider the case in which the EWSB dynamics has a global $\mathrm{SU}(2)_{L} \times \mathrm{SU}(2)_{R}$ symmetry, and imagine to fully gauge this group by enlarging the hypercharge to a whole triplet of $\mathrm{SU}(2)_{R}$. In this case the diagonal custodial $\mathrm{SU}(2)_{V}$ is exact even though $g^{\prime} \neq g$. The left and right gauge fields couple to the conserved currents of $\mathrm{SU}(2)_{L} \times \mathrm{SU}(2)_{R}$ and the interactions among two gauge fields and the Higgs boson are fully characterized in momentum space by three form factors:

$$
\left(\Gamma_{L L}\right)_{i j}^{\mu \nu}\left(p_{1}, p_{2}\right) L_{\mu}^{i} L_{\nu}^{j} h+\left(\Gamma_{L R}\right)_{i j}^{\mu \nu}\left(p_{1}, p_{2}\right) L_{\mu}^{i} R_{\nu}^{j} h+\left(\Gamma_{R R}\right)_{i j}^{\mu \nu}\left(p_{1}, p_{2}\right) R_{\mu}^{i} R_{\nu}^{j} h .
$$

Here $p_{1}, p_{2}$ are the momenta of the gauge fields and each form factor can be computed in terms of a Green function with two conserved currents, $\Gamma_{i k}^{\mu \nu}=\left\langle J_{i}^{\mu} J_{k}^{\nu} \mid h\right\rangle$. In addition to the usual massive $W$ and $Z$ bosons, which form a triplet $\hat{V}_{\mu}^{i}$ of the custodial group, in this case there is a whole triplet of massless $\mathrm{SU}(2)_{V}$ gauge fields (the photon plus its charged companion), $V_{\mu}^{i}$. The mass eigenstates $V_{\mu}$ and $\hat{V}_{\mu}$ are related to the left and right gauge fields through a rotation by an angle $\theta_{W}$, where $\tan \theta_{W}=g^{\prime} / g$. Their cubic interactions with the Higgs boson are thus characterized by three form factors, which are linear combinations of those in eq. (3.29):

$$
\begin{aligned}
& \Gamma_{V V}=\sin ^{2} \theta_{W} \Gamma_{L L}+\frac{\sin 2 \theta_{W}}{2}\left(\Gamma_{L R}+\Gamma_{R L}\right)+\cos ^{2} \theta_{W} \Gamma_{R R} \\
& \Gamma_{\hat{V} V}=\frac{\sin 2 \theta_{W}}{2} \Gamma_{L L}+\left(\cos ^{2} \theta \Gamma_{L R}-\sin ^{2} \theta \Gamma_{R L}\right)-\frac{\sin 2 \theta_{W}}{2} \Gamma_{R R} \\
& \Gamma_{\hat{V} \hat{V}}=\cos ^{2} \theta_{W} \Gamma_{L L}-\frac{\sin 2 \theta_{W}}{2}\left(\Gamma_{L R}+\Gamma_{R L}\right)+\sin ^{2} \theta_{W} \Gamma_{R R},
\end{aligned}
$$

where we have defined $\Gamma_{R L}^{\mu \nu}\left(p_{1}, p_{2}\right) \equiv \Gamma_{L R}^{\nu \mu}\left(p_{2}, p_{1}\right)$. Notice, in particular, that in this case the same form factor $\Gamma_{\hat{V} \hat{V}}$ describes the interaction of two $W$ 's and two $Z$ 's to the Higgs boson, as due to custodial invariance.

The physical limit where only $\mathrm{SU}(2)_{L} \times \mathrm{U}(1)_{Y}$ is gauged is obtained by simply switching off the unphysical $R_{\mu}^{1,2}$ fields. The interactions of two neutral vector bosons to the Higgs are still described by the relations of eq. (3.30), where $\Gamma_{Z Z}=\Gamma_{\hat{V} \hat{V}}, \Gamma_{\gamma \gamma}=\Gamma_{V V}$ and $\Gamma_{Z \gamma}=\Gamma_{\hat{V} V}$. In the charged sector, instead, the $W$ corresponds to a pure left gauge field, since it has no mixing with right-handed ones. This implies that its form factor is given by the last formula of eq. (3.30) with $\theta_{W}=0$, that is: $\Gamma_{W W}=\Gamma_{L L}$. The four physical form factors are linear combinations of the three defined in eq. (3.29), and are thus related by one identity:

$$
\begin{aligned}
\Gamma_{W W}^{\mu \nu}\left(p_{1}, p_{2}\right)-\Gamma_{Z Z}^{\mu \nu}\left(p_{1}, p_{2}\right) \cos ^{2} \theta_{W}= & \left(\Gamma_{Z \gamma}^{\mu \nu}\left(p_{1}, p_{2}\right)+\Gamma_{Z \gamma}^{\nu \mu}\left(p_{2}, p_{1}\right)\right) \frac{\sin 2 \theta_{W}}{2} \\
& +\Gamma_{\gamma \gamma}^{\mu \nu}\left(p_{1}, p_{2}\right) \sin ^{2} \theta_{W}
\end{aligned}
$$

Notice that this relation is a consequence of our initial assumption of $\mathrm{SU}(2)_{L} \times \mathrm{SU}(2)_{R}$ invariance of the EWSB dynamics. The custodial $\mathrm{SU}(2)_{V}$ is broken in this case only by the gauging of hypercharge. For $g^{\prime}=0$ the custodial symmetry is unbroken and eq. (3.31) implies $\Gamma_{W W}=\Gamma_{Z Z}$. It is straightforward to derive the relations (3.24), (3.25) and (3.28) 
from eq. (3.31). At quadratic order in the momenta, the form factors can be computed from the effective Lagrangian (3.23); one has:

$$
\begin{aligned}
\Gamma_{W W}^{\mu \nu}\left(p_{1}, p_{2}\right) & =2 m_{W}^{2} c_{W} \eta^{\mu \nu}-2 c_{W W} P_{12}^{\mu \nu}-c_{W \partial W}\left(P_{1}^{\mu \nu}+P_{2}^{\mu \nu}\right) \\
\Gamma_{Z Z}^{\mu \nu}\left(p_{1}, p_{2}\right) & =2 m_{Z}^{2} c_{Z} \eta^{\mu \nu}-2 c_{Z Z} P_{12}^{\mu \nu}-c_{Z \partial Z}\left(P_{1}^{\mu \nu}+P_{2}^{\mu \nu}\right) \\
\Gamma_{Z \gamma}^{\mu \nu}\left(p_{1}, p_{2}\right) & =-2 c_{Z \gamma} P_{12}^{\mu \nu}-c_{Z \partial \gamma} P_{2}^{\mu \nu} \\
\Gamma_{\gamma \gamma}^{\mu \nu}\left(p_{1}, p_{2}\right) & =-2 c_{\gamma \gamma} P_{12}^{\mu \nu}
\end{aligned}
$$

where we have defined $P_{1}^{\mu \nu} \equiv \eta^{\mu \nu} p_{1}^{2}-p_{1}^{\mu} p_{1}^{\nu}, P_{2}^{\mu \nu} \equiv \eta^{\mu \nu} p_{2}^{2}-p_{2}^{\mu} p_{2}^{\nu}$ and $P_{12}^{\mu \nu} \equiv \eta^{\mu \nu} p_{1} \cdot p_{2}-p_{1}^{\nu} p_{2}^{\mu}$. This is in fact the most general decomposition which follows at the $O\left(p^{2}\right)$ level for an onshell Higgs boson by assuming CP invariance and requiring that: i) the $\Gamma_{W W}, \Gamma_{Z Z}$ and $\Gamma_{\gamma \gamma}$ form factors are symmetric under the exchange $\left\{p_{1}, \mu\right\} \leftrightarrow\left\{p_{2}, \nu\right\} ;$ ii) the $\Gamma_{\gamma \gamma}$ and $\Gamma_{Z \gamma}$ form factors satisfy the Ward identities implied by $\mathrm{U}(1)_{e m}$ local invariance:

$$
p_{1 \mu} \Gamma_{\gamma \gamma}^{\mu \nu}\left(p_{1}, p_{2}\right)=0=p_{2 \nu} \Gamma_{\gamma \gamma}^{\mu \nu}\left(p_{1}, p_{2}\right), \quad p_{2 \nu} \Gamma_{Z \gamma}^{\mu \nu}\left(p_{1}, p_{2}\right)=0 .
$$

Additional structures proportional to $p_{1 \mu}$ and $p_{2 \nu}$ can be omitted since they give vanishing contributions both when the vector bosons are on-shell and when they decay into a pair of fermions by coupling to the corresponding conserved current. Inserting eq. (3.32) into (3.31) one then obtains the identities (3.24), (3.25) and (3.28).

From the above discussion it follows that if custodial symmetry is an invariance of the EWSB dynamics, the effective Lagrangians (3.23) and (2.2) have the same number of free parameters, in terms of which all observables can be computed. This is true also if one considers the fermionic operators (for a Higgs doublet these are listed in eqs. (2.3) and (2.4)), as long as one focuses on terms with one Higgs boson. This means that by using single-Higgs processes alone, one cannot distinguish the case in which the Higgs boson is part of a doublet from the more general situation. The only possible strategy to this aim is exploiting the connection among processes with zero, one and two Higgs bosons which is implied by the Lagrangian $(2.1)$ at $O\left(v^{2} / f^{2}\right)$ and does not hold in the case of the more general non-linear Lagrangian. As a consequence of such connection, the bounds that EW and flavor data set on operators with zero Higgs fields severely constrain the size of the NP effects in Higgs processes, as discussed in section (2.1). If one were to find that single-Higgs processes violate these constraints, this would be an indication that the Higgs is not part of a doublet. Furthermore, processes with double Higgs boson production can be predicted to a certain extent in terms of single-Higgs couplings, and can thus be used to probe the nature of the Higgs boson [75].

\section{Implementing the Higgs effective Lagrangian beyond the tree level}

In this section we address a few issues related to the use of the effective Lagrangians (2.1) and (3.23) beyond the tree level, as required to make Higgs precision physics without assuming the validity of the Standard Model. While the methodology is well established and various examples of its application exist in several different contexts, we think that a dedicated discussion can be useful to better clarify some specific points (see also ref. [76] 
for a recent discussion). As an illustrative though important example, we will consider the calculation of the Higgs partial decay widths, and show how the corrections from dimension6 operators can be incorporated in a consistent way. As a by-product of our analysis and to better demonstrate its applicability, in a companion paper [16] we will present a modified version of the program HDECAY $[14,15]$ that features a full implementation of the effective Lagrangian $\Delta \mathcal{L}_{S I L H}$, eq. (2.2), as well as its generalization to the case of a non-linearly realized EW symmetry, eq. (3.23).

A first difficulty which arises when using either eq. (2.1) or (3.23) is the presence of multiple expansion parameters. For generic values of the Higgs couplings $c_{i}$, the validity of the effective Lagrangian (3.23) is based on a double perturbative expansion in the SM couplings, $\alpha_{S M} / 4 \pi$, and in powers of $E / M$. The effective Lagrangian (2.1) further assumes $(v / f) \ll 1$, which implies small shifts in the Higgs couplings: $c_{i}=1+\delta c_{i}$, with $\delta c_{i} \lesssim$ $O\left(v^{2} / f^{2}\right)$. All these expansion parameters must be properly taken into account when performing calculations. Furthermore, the non-renormalizability of the effective theory implies the presence of additional divergences compared to the SM case which must be absorbed by a renormalization of the Wilson coefficients of local operators.

\subsection{RG evolution of the Wilson coefficients}

Let us discuss the issue of the renormalization and RG evolution of the Wilson coefficients first. As done in the previous sections, we will assume that the Higgs boson is part of an $\mathrm{SU}(2)_{L}$ doublet and use the Lagrangian (2.1). Since we are only interested in the divergent structure of the diagrams, it is convenient to work in the limit of unbroken $\mathrm{SU}(2)_{L} \times \mathrm{U}(1)_{Y}$ and compute the Green functions in terms of the Higgs doublet $H$. The only 1-loop diagrams which generate additional logarithmic divergences are those featuring one insertion of the effective vertices from dimension-6 operators. By dimensional analysis, further insertions of the effective vertices lead to power-divergent contributions to dimension- 6 operators (which are irrelevant to determine the RG running) and log-divergent contributions to higher-dimensional operators. The same counting holds also at higher loop level: the only log-divergent contribution to dimension-6 operators comes from diagrams with one insertion of the effective couplings, and is thus suppressed by extra powers of the SM expansion parameter $\alpha_{S M} / 4 \pi$. This is in analogy with the renormalization of the pion effective Lagrangian in the chiral limit, see ref. [77]. It thus follows that the RG equation is linear and homogeneous in the $\bar{c}_{i}$, and different operators with the same quantum numbers will in general mix with each other. At leading order in $\alpha_{S M}$, with $\alpha_{S M}=\alpha_{e m}, \alpha_{2}, \alpha_{s}$, respectively, in the case of electromagnetic, weak and QCD corrections, one has

$$
\bar{c}_{i}(\mu)=\left(\delta_{i j}+\gamma_{i j}^{(0)} \frac{\alpha_{S M}(\mu)}{4 \pi} \log \left(\frac{\mu}{M}\right)\right) \bar{c}_{j}(M),
$$

where $\gamma_{i j}^{(0)}$ is the leading-order coefficient of the anomalous dimension. Some elements of the anomalous dimension matrix $\gamma_{i j}^{(0)}$ have been recently computed in refs. [78, 79].

In the case in which the Higgs boson and possibly the SM quarks (in particular the top and the bottom) are strongly coupled to the new dynamics, the leading RG running effect comes from loops of these particles and can be as large as $\Delta \bar{c}_{i} / \bar{c}_{i}(M) \sim$ 


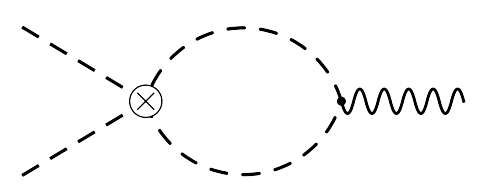

(a)

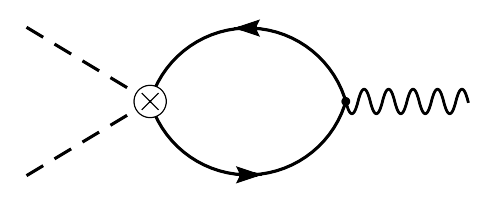

(b)

Figure 1. One-loop diagrams relevant for the RG running of $\bar{c}_{W}$ and $\bar{c}_{B}$. Dashed, continuous and wiggly lines denote, respectively, a weak doublet $H$, a fermion and a vector field $V=W, B$. The symbol $\otimes$ denotes the insertion of the effective vertex from $O_{H}$ (in diagram $(a)$ ) or $O_{H \psi}$ (in diagram $(b))$.

$\left(g_{*}^{2} / 16 \pi^{2}\right) \log (M / \mu)$ or $\left(\lambda_{\psi}^{2} / 16 \pi^{2}\right) \log (M / \mu)$. This must be compared to the effects of order $\left(g_{S M}^{2} / 16 \pi^{2}\right) \log (M / \mu)$ from loops of gauge fields. For example, the insertion of $\bar{c}_{H}$ in the diagram (a) of figure 1 leads to a renormalization of $O_{W+B} \equiv O_{W}+O_{B}$ :

$$
\bar{c}_{W+B}(\mu)=\bar{c}_{W+B}(M)-\frac{1}{6} \frac{\alpha_{2}}{4 \pi} \log \left(\frac{\mu}{M}\right) \bar{c}_{H}(M),
$$

where $\alpha_{2}$ has been defined in eq. (3.20). It is well known that this RG running is associated with the IR contribution to the $\epsilon_{3}$ parameter, and the same coefficient $\gamma_{W+B, H}^{(0)}=-1 / 6$ can indeed be extracted from self-energy diagrams [80]. From the estimates of eq. (2.9), $\bar{c}_{H}(M) \sim O\left(v^{2} / f^{2}\right), \bar{c}_{W, B}(M) \sim O\left(m_{W}^{2} / M^{2}\right)$, it follows that the correction to $\bar{c}_{W+B}$ from its RG evolution down to the scale $\mu$ is of order $\Delta \bar{c}_{W+B} / \bar{c}_{W+B}(M) \sim\left(g_{*}^{2} / 16 \pi^{2}\right) \log (M / \mu)$ as anticipated. Similarly, the insertion of $\bar{c}_{H \psi}$ into a loop of fermions, like in diagram $(b)$ of figure 1 , leads to a renormalization of $\bar{c}_{W}$ and $\bar{c}_{B}$ :

$$
\Delta \bar{c}_{W, B} \approx N_{c} \frac{\alpha_{2}}{4 \pi} \log \left(\frac{\mu}{M}\right) \bar{c}_{H \psi}(M),
$$

where $N_{c}=3$ is a color factor. In this case the RG correction is of order $\left(\lambda_{\psi}^{2} / 16 \pi^{2}\right) \log (M / \mu)$ compared to the UV value of the coefficients, as one can immediately verify by using the estimates (2.9).

Loops of EW gauge fields give corrections which are suppressed by a weak loop factor $\left(g^{2} / 16 \pi^{2}\right)$, and the associated RG evolution is therefore generically small. An important exception is the case in which the Wilson coefficient has a value suppressed at the scale $M$. For example, if the dynamics behind the EW symmetry breaking is custodially invariant, then $\bar{c}_{T}(M)=0$. The insertion of $\bar{c}_{H}$ into a loop of hypercharge gauge bosons, as in diagram (a) of figure 2, renormalizes $\bar{c}_{T}$ and gives

$$
\bar{c}_{T}(\mu)=\frac{3}{2} \tan ^{2} \theta_{W} \frac{\alpha_{2}}{4 \pi} \log \left(\frac{\mu}{M}\right) \bar{c}_{H}(M) .
$$

Compared to the naive estimate of eq. $(2.9), \bar{c}_{T}(M) \sim O\left(v^{2} / f^{2}\right)$, valid in absence of custodial symmetry, the above correction is further suppressed by a factor $\left(g^{2} / 16 \pi^{2}\right) \log (M / \mu)$. Although small, such a low-energy value of $\bar{c}_{T}$ has a strong impact on the EW precision tests performed at LEP [80]. ${ }^{18}$ On the other hand, it is too small to be observable through

\footnotetext{
${ }^{18}$ For example, $\bar{c}_{T}\left(m_{Z}\right) \sim 10^{-3}$ for $\bar{c}_{H}(M) \sim 0.1$.
} 


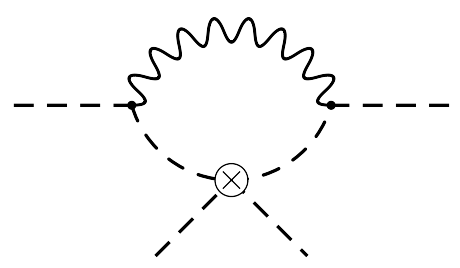

(a)

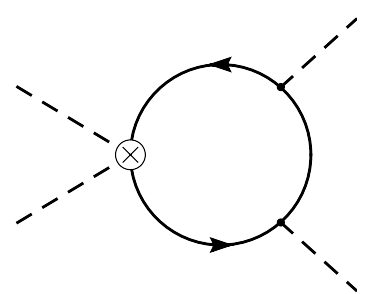

(b)

Figure 2. One-loop diagrams relevant for the RG running of $\bar{c}_{T}$. Dashed, continuous and wiggly lines denote, respectively, a weak doublet $H$, a fermion and a hypercharge field $B$. The symbol $\otimes$ denotes the insertion of the effective vertex from $O_{H}$ (in diagram $(a)$ ) or $O_{H \psi}$ (in diagram $(b)$ ).

a measurement of the Higgs couplings at the LHC. A similar renormalization of $\bar{c}_{T}$ also follows from loops of SM fermions through the insertion of $\bar{c}_{H \psi}$, as illustrated by diagram (b) of figure 2. The explicit calculation for the case of a composite right- and left-handed top quark was performed for example in ref. [81]. Naively, the effect goes like

$$
\Delta \bar{c}_{T} \approx N_{c} \frac{y_{\psi}^{2}}{16 \pi^{2}} \log \left(\frac{\mu}{M}\right) \bar{c}_{H \psi}(M)
$$

and is of order $\left(y_{\psi} / g^{\prime}\right)^{2}\left(\lambda_{\psi} / g_{*}\right)^{2}$ compared to the one from loops of hypercharge. ${ }^{19}$

In general, although small, the RG evolution of the Wilson coefficients due to EW loops must be properly taken into account in order to precisely match the experimental results obtained at low energy with the theory predictions at high energy. This is even more true in the case of QCD loop corrections, which can be large and will affect the coefficients of the dimension- 6 operators with quarks and gluon fields. ${ }^{20}$ The effect of the running of the Wilson coefficients can be easily incorporated in programs for the automatic calculation of production cross sections and decay rates by using the effective Lagrangian (2.1) and identifying the coefficients appearing there as their values at the relevant low-energy scale.

\subsection{Decay rates at the loop level with the effective Lagrangian}

In addition to the short-distance effects discussed above, which are parametrized in terms of the evolution of the coefficients of local operators, one-loop diagrams also lead to long-distance corrections to the observables under consideration. Specifically, while shortdistance effects are related to the divergent terms, the long-distance contributions correspond to the finite parts and are defined in a given renormalization scheme. In general,

\footnotetext{
${ }^{19}$ Notice that in case of a sizable fermion coupling $\lambda_{\psi}$, a numerically larger contribution to $\bar{c}_{T}$ comes from fermionic loops with two insertions of $\bar{c}_{H \psi}$. The corresponding diagram is quadratically divergent, so that it gives a threshold correction to $\bar{c}_{T}$ at the scale $M$, but does not contribute to its running. An explicit calculation can be found in ref. [81] for the case of a composite top quark. Naively the effect is of order $\Delta \bar{c}_{T} \sim N_{c}(v / f)^{2}\left(\lambda_{\psi} / 16 \pi^{2}\right)\left(\lambda_{\psi} / g_{*}\right)^{2}$, and can be numerically large. For example, if both $t_{L}$ and $t_{R}$ couple with the same strength $\lambda_{t_{L}}=\lambda_{t_{R}} \sim \sqrt{g_{*} y_{t}}$ to the new dynamics, then it follows $\Delta \bar{c}_{T} \sim N_{c}(v / f)^{2}\left(y_{t}^{2} / 16 \pi^{2}\right)$.

${ }^{20}$ Notice that $g_{s}^{2} \bar{c}_{g}$ is not renormalized at one-loop by QCD corrections. This follows from the RGinvariance of the operator $\left(\beta\left(g_{s}\right) / g_{s}\right) G_{\mu \nu} G^{\mu \nu}$ which contributes to the trace of the energy-momentum tensor [82-84]. See also the recent discussion in ref. [78].
} 
the decay amplitude can be expanded as follows: ${ }^{21}$

$$
A=A_{0}^{S M}+A_{1}^{S M}+\Delta A_{0}+\Delta A_{1}+\ldots
$$

where $A_{0}^{S M}\left(A_{1}^{S M}\right)$ is the tree-level (one-loop) SM amplitude, and $\Delta A_{0}\left(\Delta A_{1}\right)$ is the treelevel (one-loop) contribution from the dimension-6 operators of the effective Lagrangian in eqs. (2.2)-(2.4). The dots denote higher-loop contributions as well as the corrections due to higher-order operators.

Let us consider for example the decay $h \rightarrow W^{(*)} W^{*}$. In this case the operators that can contribute at tree-level are $O_{H}, O_{W}, O_{H W}, O_{\psi W}, O_{H \psi}^{\prime}$, as well as $O_{H u d}$ in the case in which the off-shell $W$ decays into a pair of quarks. Based on the naive estimates of eq. (2.9) and according to the discussion of section 3 , we can quantify the various effects encoded by $\Delta A_{0}$ as follows:

$$
\begin{aligned}
\frac{\Delta A_{0}}{A_{0}^{S M}}\left(W^{(*)} W^{*}\right)= & \hat{c}_{H} \times O\left(\frac{v^{2}}{f^{2}}\right)+\hat{c}_{W} \times O\left(\frac{E^{2}}{M^{2}}\right)+\hat{c}_{H W} \times O\left(\frac{E^{2}}{16 \pi^{2} f^{2}}\right) \\
& +\hat{c}_{H u d} \times O\left(\frac{v^{2}}{f^{2}} \frac{\lambda_{u} \lambda_{d}}{g_{*}^{2}}\right)+\hat{c}_{H \psi}^{\prime} \times O\left(\frac{v^{2}}{f^{2}} \frac{\lambda_{\psi}^{2}}{g_{*}^{2}}\right)+\hat{c}_{\psi W} \times O\left(\frac{E m_{\psi}}{16 \pi^{2} f^{2}}\right) .
\end{aligned}
$$

Here $E=m_{h}$ is the relevant energy of the process and we have conveniently defined each of the $O(1)$ parameters $\hat{c}_{i}$ to be equal to $\bar{c}_{i}\left(m_{h}\right)$ divided by its naive estimate in eq. (2.9):

$$
\begin{aligned}
& \hat{c}_{i}=\frac{f^{2}}{v^{2}} \bar{c}_{i}\left(m_{h}\right), \quad i=H, T, 6, \psi, \quad \hat{c}_{i}=\frac{M^{2}}{m_{W}^{2}} \bar{c}_{i}\left(m_{h}\right), \quad i=W, B, \\
& \hat{c}_{i}=\frac{16 \pi^{2} f^{2}}{m_{W}^{2}} \bar{c}_{i}\left(m_{h}\right), \quad i=H W, H B, \gamma, g, \psi W, \psi B, \psi G, \\
& \hat{c}_{i}=\frac{g_{*}^{2}}{\lambda_{\psi}^{2}} \frac{f^{2}}{v^{2}} \bar{c}_{i}\left(m_{h}\right), \quad \hat{c}_{i}^{\prime}=\frac{g_{*}^{2}}{\lambda_{\psi}^{2}} \frac{f^{2}}{v^{2}} \bar{c}_{i}^{\prime}\left(m_{h}\right), \quad i=H \psi, \quad \hat{c}_{H u d}=\frac{g_{*}^{2}}{\lambda_{u} \lambda_{d}} \frac{f^{2}}{v^{2}} \bar{c}_{H u d}\left(m_{h}\right) .
\end{aligned}
$$

When the Higgs boson is pNGB, the two parameters $\hat{c}_{g}$ and $\hat{c}_{\gamma}$ are not of order one but are further suppressed by a factor $g_{G}^{2} / g_{*}^{2}$. From eq. (4.7) one can see that the contribution of the dipole operators $O_{\psi W}$ is suppressed by $\left(m_{\psi} / m_{h}\right)$ compared to that of $O_{H W}$, while that of $O_{H u d}$ and $O_{H \psi}^{\prime}$ is expected to be small given the existing constraints on the couplings $\lambda_{\psi}$ (see the discussion in section 2.1). The dominant NP contribution thus comes from the terms in the first line of eq. (4.7), among which the one proportional to $\bar{c}_{H}$ is the leading effect for $g_{*}>g$. The 1-loop electroweak amplitude $A_{1}^{S M}$ gives a contribution of order $A_{1}^{S M} / A_{0}^{S M} \sim\left(\alpha_{2} / 4 \pi\right)$. We thus see explicitly that $\Delta A_{0}$ and $A_{1}^{S M}$ encode the NLO corrections in the three expansion parameters which we are considering: $\alpha_{2} / 4 \pi$ (electroweak

\footnotetext{
${ }^{21}$ In the strict sense this equation is valid for the genuine EW corrections only, while for simplicity we include the (IR-divergent) virtual QED corrections to the SM amplitude in the same way. The corresponding real photon radiation contributions to the decay rates are treated in terms of a linear novel contribution to the Higgs coupling for the squared amplitude in order to obtain an infrared finite result. Pure QED corrections factorize as QCD corrections in general so that their amplitudes scale with the modified Higgs couplings. However, they cannot be separated from the genuine EW corrections in a simple way.
} 
expansion), $E^{2} / M^{2}$ (derivative expansion) and $v^{2} / f^{2}$. The contribution due to 1-loop diagrams with one insertion of the effective vertices has not been computed yet, but we can easily estimate its size:

$$
\frac{\Delta A_{1}}{A_{0}^{S M}}\left(W^{(*)} W^{*}\right)=\hat{c}_{H} \times O\left(\frac{v^{2}}{f^{2}} \frac{\alpha_{2}}{4 \pi}\right)+\hat{c}_{u} \times O\left(\frac{v^{2}}{f^{2}} \frac{\alpha_{2}}{4 \pi}\right)+\hat{c}_{6} \times O\left(\frac{v^{2}}{f^{2}} \frac{\alpha_{2}}{4 \pi}\right)+\ldots
$$

where the dots denote the subleading terms due to the other operators. The terms shown in eq. (4.9) arise from the same 1-loop diagrams that give the SM amplitude $A_{1}^{S M}$, where each of the Higgs couplings gets shifted by $\bar{c}_{H}, \bar{c}_{u}$ and $\bar{c}_{6}$. By neglecting the unknown $\Delta A_{1}$ one is omitting terms of order $\left(v^{2} / f^{2}\right)\left(\alpha_{2} / 4 \pi\right)$, that is, of the same size of the tree-level contribution due to the operator $O_{H W}$, see eq. (4.7), since $E=m_{h} \approx m_{W}$. This latter contribution can be easily computed and it is included in the formula of the decay rate to $W W$ (and similarly that of $O_{H W}$ and $O_{H B}$ to $Z Z$ is also included) implemented in the program eHDECAY discussed in ref. [16]. The addition of the tree-level correction from $O_{H W}$ is clearly the first step towards a full inclusion of the $O\left[\left(v^{2} / f^{2}\right)\left(\alpha_{2} / 4 \pi\right)\right]$ corrections, where the missing part will have to be computed from 1-loop diagrams featuring one insertion of $O_{H}, O_{u}$ and $O_{6}$. It is worth noting that these diagrams, in general, contain logarithmic divergences which must be reabsorbed by a renormalization of the Wilson coefficients and contribute to their RG evolution as explained in the previous section. The finite part is the contribution to $\Delta A_{1}$ which awaits to be computed.

By approximating the amplitude as $A \simeq A_{0}^{S M}+A_{1}^{S M}+\Delta A_{0}$ one obtains the following formula for the decay rate: ${ }^{22}$

$$
\begin{aligned}
\Gamma\left(W^{(*)} W^{*}\right)=\Gamma_{0}^{S M}\left(W^{(*)} W^{*}\right)\{1 & +\frac{2}{\left|A_{0}^{S M}\right|^{2}} \operatorname{Re}\left[\left(A_{0}^{S M}\right)^{*}\left(A_{1}^{S M}+\Delta A_{0}\right)\right] \\
& \left.+O\left(\left(\frac{v^{2}}{f^{2}}\right)^{2},\left(\frac{\alpha_{2}}{4 \pi} \frac{v^{2}}{f^{2}}\right),\left(\frac{\alpha_{2}}{4 \pi}\right)^{2}\right)\right\}
\end{aligned}
$$

where $\Gamma_{0}^{S M}\left(W^{(*)} W^{*}\right)$ denotes the tree-level SM decay rate. For simplicity, we have not shown terms involving powers of $E^{2} / M^{2}$ among the neglected contributions, since for $E=m_{h} \approx m_{W}$ one has $E^{2} / M^{2} \lesssim v^{2} / f^{2}$ if $g_{*} \gtrsim g$. As mentioned, this formula incorporates the $O\left(v^{2} / f^{2}\right), O\left(\alpha_{2} / 4 \pi\right)$ and $O\left(m_{h}^{2} / M^{2}\right)$ corrections (NLO in the perturbative expansion), and can be easily implemented in existing codes for the automatic computation of the decay rate. The inclusion of the $O\left(m_{h}^{2} / M^{2}\right)$ tree-level correction due to $O_{W}$ is justified as long as $g_{*}<4 \pi$, since it is parametrically larger than the neglected $O\left[\left(v^{2} / f^{2}\right)\left(\alpha_{2} / 4 \pi\right)\right]$ terms by a factor $\left(16 \pi^{2} / g_{*}^{2}\right)$. Notice that in the limit of large deviations of the Higgs couplings from their SM values, $(v / f)^{2} \sim O(1)$, the neglected terms of $O\left[\left(v^{2} / f^{2}\right)\left(\alpha_{2} / 4 \pi\right)\right]$ become as important as those included through $A_{1}^{S M}$. In other words, a proper inclusion of the EW corrections in the limit $v \sim f$ requires a complete 1-loop calculation where each of the diagrams is rescaled by the appropriate coupling factor.

\footnotetext{
${ }^{22}$ The same remark as in footnote 21 applies.
} 
A similar discussion applies to the Higgs decay into a pair of fermions, $h \rightarrow \bar{\psi} \psi$. In this case only $O_{H}$ and $O_{\psi}(\psi=u, d, l)$ contribute at tree level,

$$
\frac{\Delta A_{0}}{A_{0}^{S M}}(\bar{\psi} \psi)=\left(\frac{\hat{c}_{H}}{2}+\hat{c}_{\psi}\right) \times O\left(\frac{v^{2}}{f^{2}}\right)
$$

while the one-loop EW diagrams featuring one effective vertex give a correction of order

$$
\frac{\Delta A_{1}}{A_{0}^{S M}}(\bar{\psi} \psi)=\hat{c}_{H} \times O\left(\frac{v^{2}}{f^{2}} \frac{\alpha_{2}}{4 \pi}\right)+\hat{c}_{\psi} \times O\left(\frac{v^{2}}{f^{2}} \frac{\alpha_{2}}{4 \pi}\right)+\hat{c}_{6} \times O\left(\frac{v^{2}}{f^{2}} \frac{\alpha_{2}}{4 \pi}\right)+\ldots
$$

where the dots indicate the subleading terms due to the other operators. The calculation of $\Delta A_{1}$ has not been performed yet, while the 1-loop EW corrections are known in the SM, $A_{1}^{S M}$. Their inclusion is thus possible as long as $(v / f) \ll 1$, so that the neglected terms in $\Delta A_{1}$ are subleading. The case of $\mathrm{QCD}$ radiative corrections is different, since at leading order they factorize with respect to the expansion in the number of derivative and fields and can thus be resummed up to higher orders. In the case of the Higgs decay into a pair of quarks one can for example approximate $A \simeq A_{0}^{S M}+A_{1}^{S M}+\Delta A_{0}$ and obtain the following formula for the decay rate: ${ }^{23}$

$$
\begin{aligned}
\Gamma(\bar{q} q)=\Gamma_{0}^{S M}(\bar{q} q) \kappa^{Q C D}\{1 & +\frac{2}{\left|A_{0}^{S M}\right|^{2}} \operatorname{Re}\left[\left(A_{0}^{S M}\right)^{*}\left(A_{1}^{S M}+\Delta A_{0}\right)\right] \\
& \left.+O\left(\left(\frac{v^{2}}{f^{2}}\right)^{2},\left(\frac{\alpha_{2}}{4 \pi} \frac{v^{2}}{f^{2}}\right),\left(\frac{\alpha_{2}}{4 \pi}\right)^{2}\right)\right\}
\end{aligned}
$$

where $\Gamma_{0}^{S M}(\bar{q} q)$ is the SM tree-level rate and $\kappa^{Q C D}$ encodes the QCD corrections. This formula includes the leading $O\left(v^{2} / f^{2}\right), O\left(\alpha_{2} / 4 \pi\right)$ and QCD corrections. Mixed electroweak and QCD corrections can also be included by assuming that they factorize, as the nonfactorizable terms are known to be small. Compared to the decay rate into $W W$, eq. (4.13) apparently does not include corrections of order $m_{h}^{2} / M^{2}$. While there is indeed no operator whose contribution starts at that order, such corrections can arise from subleading contributions to $\bar{c}_{H}$ and $\bar{c}_{\psi}$. For example, the tree-level exchange of heavy fermions can lead to a wave-function renormalization of the SM ones, which can be re-expressed in our notation as a contribution to $\bar{c}_{\psi}$ of order $\lambda_{\psi}^{2} v^{2} / M^{2}$.

A similar resummation of the QCD corrections also works for the decay $h \rightarrow g g$. In this case the SM tree-level amplitude vanishes, $A_{0}^{S M}=0$, while the leading contribution arises from the 1-loop exchange of top quarks. The two-loop EW corrections are known in the SM and give a correction of order $A_{2}^{S M} / A_{1}^{S M} \sim \alpha_{2} / 4 \pi$. Among the dimension-6 operators, only $O_{g}$ contributes at tree-level,

$$
\frac{\Delta A_{0}}{A_{1}^{S M}}(g g)=\hat{c}_{g} \times O\left(\frac{v^{2}}{f^{2}}\right) .
$$

As discussed in section 2 (see eq. (2.10)), the above estimate is suppressed by an additional factor $\left(g_{G}^{2} / g_{*}^{2}\right)$ in the case of a NG Higgs boson. At the one-loop level one has

$$
\frac{\Delta A_{1}}{A_{1}^{S M}}(g g)=\left(\frac{\hat{c}_{H}}{2}+\hat{c}_{u}\right) \times O\left(\frac{v^{2}}{f^{2}}\right)+\hat{c}_{t G} \times O\left(\frac{v^{2}}{f^{2}} \frac{y_{t}^{2}}{16 \pi^{2}}\right) .
$$

\footnotetext{
${ }^{23}$ The same remark as in footnote 21 applies.
} 
Thus, the one-loop effect of $O_{H}$ and $O_{u}$ is expected to be as important as the tree-level one from $O_{g}$, and even larger if the Higgs is a NG boson, as discussed in section 3.3. This is in fact not surprising, since $\bar{c}_{g}$ arises at the 1-loop level in minimally coupled theories, while $\bar{c}_{H}$ and $\bar{c}_{u}$ can be generated at tree level. The contribution from the dipole operator $O_{t G}$ is suppressed by a factor $y_{t}^{2} / 16 \pi^{2}$ compared to that from $O_{H}$ and $O_{u}$, as expected from the fact that $\bar{c}_{t G}$ is generated at the 1-loop level in minimally coupled theories. For this reason it can be neglected. It should be noted that without a complete computation of the NLO EW corrections of order $\left(\alpha_{2} / 4 \pi\right)\left(v^{2} / f^{2}\right)$, the LHC data on Higgs physics are not sensitive to the range of values of $\bar{c}_{t G}$ expected using the naive estimate (2.9) with $(v / f)^{2} \sim 0.1$. Furthermore, we stress that in order to distinguish the effect of $O_{t G}$ from that of $O_{g}$, the $t \bar{t} h$ channel should be measured [69] (single top production in association with the Higgs could also provide complementary information [85]). Also in this case, there are no operators giving $m_{h}^{2} / M^{2}$ corrections, although these terms will in general appear as subleading contributions to $\bar{c}_{g}, \bar{c}_{H}$ and $\bar{c}_{u}$, as discussed above. It is well known that higher-order $\alpha_{s}$ corrections are large, so they must be included consistently in our perturbative expansion. This can be done easily in the approximation $m_{h} \ll 2 m_{t}$, which is reasonably accurate for $m_{h}=125 \mathrm{GeV}$. In such a limit one can integrate out the top quark and match its one-loop contribution to that of the local operator $O_{g}$. Then it trivially follows that the QCD corrections associated to the virtual exchange and real emissions of gluons and light quarks below the scale $m_{t}$ factorize in the rate, the multiplicative factor being the same for both the top quark and New Physics terms. By approximating $A \simeq$ $A_{1}^{S M}+A_{2}^{S M}+\Delta A_{0}+\Delta A_{1}$, one arrives at the following formula for the $h \rightarrow g g$ decay rate:

$$
\begin{gathered}
\Gamma(g g)=\Gamma_{1}^{S M}(g g) \kappa_{\text {soft }}\left\{c_{\mathrm{eff}}^{2}+\frac{2 c_{\mathrm{eff}}}{\left|A_{1}^{S M}\right|^{2}} \operatorname{Re}\left[\left(A_{1}^{S M}\right)^{*}\left(A_{2}^{S M} c_{\mathrm{eff}}+\Delta A_{0}+\Delta A_{1} c_{\mathrm{eff}}\right)\right]\right. \\
\left.+O\left(\left(\frac{v^{2}}{f^{2}}\right)^{2},\left(\frac{\alpha_{2}}{4 \pi} \frac{v^{2}}{f^{2}}\right),\left(\frac{\alpha_{2}}{4 \pi}\right)^{2}\right)\right\},
\end{gathered}
$$

where $\Gamma_{1}^{S M}(g g)$ is the 1-loop SM decay width. The factor $c_{\mathrm{eff}}$ includes all the dependence on $m_{t}$ and accounts for virtual QCD corrections to $A_{1}^{S M}$ above that scale, while $\kappa_{\text {soft }}$ parametrizes the soft radiative effects. By using eq. (4.16), the existing four-loop calculations of $c_{\text {eff }}[86-89]$ and $\kappa_{\text {soft }}$ [90-94] allow one to include the QCD corrections up to $\mathrm{N}^{3} \mathrm{LO}$.

The contributions to the decay $h \rightarrow \gamma \gamma$ follow a similar pattern as for $h \rightarrow g g$. At tree level:

$$
\frac{\Delta A_{0}}{A_{1}^{S M}}(\gamma \gamma)=\hat{c}_{\gamma} \times O\left(\frac{v^{2}}{f^{2}}\right)
$$

At one loop:

$$
\begin{aligned}
\frac{\Delta A_{1}}{A_{1}^{S M}}(\gamma \gamma)= & \hat{c}_{H} \times O\left(\frac{v^{2}}{f^{2}}\right)+\hat{c}_{u} \times O\left(\frac{v^{2}}{f^{2}}\right)+\hat{c}_{W} \times O\left(\frac{m_{W}^{2}}{M^{2}}\right) \\
& +\hat{c}_{H W} \times O\left(\frac{m_{W}^{2}}{16 \pi^{2} f^{2}}\right)+\left(\hat{c}_{t W}+\hat{c}_{t B}\right) \times O\left(\frac{v^{2}}{f^{2}} \frac{y_{t}^{2}}{16 \pi^{2}}\right) .
\end{aligned}
$$

The 2-loop electroweak corrections have been computed in the SM and can be included for $\left(v^{2} / f^{2}\right) \ll 1$, so that unknown $O\left[\left(v^{2} / f^{2}\right)\left(\alpha_{2} / 4 \pi\right)\right]$ effects arising from 2-loop diagrams with 
one effective vertex are negligible. From eq. (4.18) one can see that the 1-loop contribution due to $O_{H W}$ is of the same order as such neglected terms. The 1-loop correction from $O_{W}$, on the contrary, is parametrically larger by a factor $\left(16 \pi^{2} / g_{*}^{2}\right)$ and should be included for $g_{*}<4 \pi$. The easiest way to compute it is by rewriting $O_{W}$ in terms of the other operators through the equations of motion [4], see eq. (3.8). The 1-loop correction due to the dipole operators is suppressed by a factor $y_{t}^{2} / 16 \pi^{2}$ and can be neglected. Approximating $A \simeq A_{1}^{S M}+A_{2}^{S M}+\Delta A_{0}+\Delta A_{1}$ one finds:

$$
\begin{aligned}
\Gamma(\gamma \gamma)=\Gamma_{1}^{S M}(\gamma \gamma)\{1 & +\frac{2}{\left|A_{1}^{S M}\right|^{2}} \operatorname{Re}\left[\left(A_{1}^{S M}\right)^{*}\left(A_{2}^{S M}+\Delta A_{0}+\Delta A_{1}\right)\right] \\
& \left.+O\left(\left(\frac{v^{2}}{f^{2}}\right)^{2},\left(\frac{\alpha_{2}}{4 \pi} \frac{v^{2}}{f^{2}}\right),\left(\frac{\alpha_{2}}{4 \pi}\right)^{2}\right)\right\}
\end{aligned}
$$

Finally, the estimate of the corrections to $h \rightarrow \gamma Z$ is the following:

$$
\begin{aligned}
& \frac{\Delta A_{0}}{A_{1}^{S M}}(Z \gamma)=\hat{c}_{\gamma} \times O\left(\frac{v^{2}}{f^{2}}\right)+\left(\hat{c}_{H W}-\hat{c}_{H B}\right) \times O\left(\frac{v^{2}}{f^{2}}\right), \\
& \frac{\Delta A_{1}}{A_{1}^{S M}}(Z \gamma)=\hat{c}_{H} \times O\left(\frac{v^{2}}{f^{2}}\right)+\hat{c}_{u} \times O\left(\frac{v^{2}}{f^{2}}\right)+\hat{c}_{W} \times O\left(\frac{m_{W}^{2}}{M^{2}}\right)+\hat{c}_{H W} \times O\left(\frac{m_{W}^{2}}{16 \pi^{2} f^{2}}\right) \\
& +\hat{c}_{t W} \times O\left(\frac{v^{2}}{f^{2}} \frac{y_{t}^{2}}{16 \pi^{2}}\right)+\hat{c}_{t B} \times O\left(\frac{v^{2}}{f^{2}} \frac{y_{t}^{2}}{16 \pi^{2}}\right) .
\end{aligned}
$$

In this case the 1-loop electroweak corrections are not known in the SM, so that the formula for the decay rate reads:

$$
\Gamma(Z \gamma)=\Gamma_{1}^{S M}(Z \gamma)\left\{1+\frac{2}{\left|A_{1}^{S M}\right|^{2}} \operatorname{Re}\left[\left(A_{1}^{S M}\right)^{*}\left(\Delta A_{0}+\Delta A_{1}\right)\right]+O\left(\left(\frac{v^{2}}{f^{2}}\right)^{2},\left(\frac{\alpha_{2}}{4 \pi}\right)\right)\right\}
$$

where only the contributions from $O_{H}, O_{u}$ and $O_{W}$ should be retained in $\Delta A_{1}$ for consistency.

Through the above discussion we sketched how the effective Lagrangian can be implemented beyond the tree level in the calculation of physical quantities. In the case of the Higgs partial decay widths, in particular, we have seen how the EW and QCD corrections can be included consistently with the expansion in the number of fields and derivatives. As a more concrete illustration of these considerations, we have written a modified version of the program HDECAY, which we dub eHDECAY, where the corrections from all the local operators of the effective Lagrangians (2.2) and (3.23) are included at NLO. A detailed description of the code is given in ref. [16], where more explicit formulas for each of the Higgs partial widths are provided.

\section{Discussion}

The discovery of a resonance with a mass around $125 \mathrm{GeV}$ similar to the long-sought Standard Model Higgs boson brings the exploration of the electroweak symmetry breaking 
sector under quantitative scrutiny. The LHC experiments, together with those at the Tevatron, report the signal strengths, i.e. the product of the Higgs production cross section times its decay branching ratio, for various final state channels. The main task of the community is now to interpret these data and understand the implications for the theory of New Physics that is expected to lie beyond the weak scale.

The EW oblique parameters provide a bound on the scale of New Physics but do not give detailed information about the nature of the NP sector. In order to understand how the weak scale is stabilized at the quantum level, i.e. how the hierarchy problem is solved, one crucial question is whether EW symmetry breaking proceeds by weak or strong dynamics. The direct observation of new degrees of freedom would provide a straightforward answer. But a glimpse of New Physics can also be caught from a dedicated study of the Higgs boson itself, and in particular from a measurement of its couplings, if a departure from the SM predictions is ever observed. It is useful to parametrize the deviations from the SM by the effective Lagrangian of eq. (2.1). By measuring its Wilson coefficients $\bar{c}_{i}$ one can infer what kind of UV theory completes the SM.

If the coupling strength of the Higgs boson to the NP sector is of the order of the SM weak couplings, $g_{*} \approx g$, then our power counting (2.9) shows that the coefficients of the operators that can be generated at tree-level, $O_{H}, O_{u, d, l}, O_{W}$ and $O_{B}$, are expected to be all of the same order, $m_{W}^{2} / M^{2}$, where $M$ is the typical mass scale of the NP spectrum, unless some special selection rule suppresses some of them. It is instructive to examine the predictions of the archetypal example of weakly-coupled UV completions: the Minimal Supersymmetric Standard Model (MSSM). First, $R$-parity protects the EW oblique parameters from any tree-level contributions, hence $\bar{c}_{W}$ and $\bar{c}_{B}$ are of order $\left(m_{W}^{2} / M^{2}\right)\left(\alpha_{2} / 4 \pi\right)$ and thus small. Second, the couplings of the lightest Higgs boson to the massive gauge bosons are given by $c_{V}=\sin (\beta-\alpha)$, where $\alpha$ is the rotation angle to diagonalize the CPeven mass matrix and $\tan \beta$ is the ratio of the vacuum expectation values of the two neutral CP-even Higgs bosons. In the decoupling limit, $\alpha \rightarrow \beta-\pi / 2$, one has $c_{V}=1+O\left(m_{Z}^{4} / m_{H}^{4}\right)$, where $m_{H}$ is the mass of the heaviest CP-even scalar (for a general treatment of the decoupling limit see for example ref. [95]). This means that at tree-level the deviations of the Higgs-gauge boson couplings are generated by dimension- 8 operators [96], while $\bar{c}_{H}$ arises only through loop effects and is naively of order $\left(m_{W}^{2} / M^{2}\right)\left(\alpha_{2} / 4 \pi\right)$. At the same time, the couplings to up- and down-type quarks read, respectively,

$$
\begin{aligned}
& c_{u}=+\frac{\cos \alpha}{\sin \beta}=1+2 \frac{m_{Z}^{2}}{m_{H}^{2}} \cos ^{2} \beta \cos 2 \beta+O\left(\frac{m_{Z}^{4}}{m_{H}^{4}}\right) \\
& c_{d}=-\frac{\sin \alpha}{\cos \beta}=1-2 \frac{m_{Z}^{2}}{m_{H}^{2}} \sin ^{2} \beta \cos 2 \beta+O\left(\frac{m_{Z}^{4}}{m_{H}^{4}}\right) .
\end{aligned}
$$

For moderately large $\tan \beta$ this implies $\bar{c}_{d} \sim m_{Z}^{2} / m_{H}^{2}$, while $\bar{c}_{u}$ is further suppressed by a factor $\sim 1 / \tan ^{2} \beta$ (see for example refs. [97, 98] and the recent discussion in ref. [99]). A pattern with small values of $\bar{c}_{H}, \bar{c}_{W}, \bar{c}_{B}$ and $\bar{c}_{u}$ but with a $\sim 15 \%$ enhancement of the Higgs coupling to down-type quarks due to $\bar{c}_{d}$, for example, would be indicative of the MSSM with large $\tan \beta$ and the additional Higgs bosons around $300 \mathrm{GeV}$. Generic twoHiggs doublet models lead to a similar pattern of couplings, while models where the Higgs 
boson mixes with a scalar that is singlet under the SM gauge group can generate $\bar{c}_{H}$ at the tree level. In the MSSM, loops of light stops or staus as well as charginos can also give sizable contributions to the effective couplings of the light Higgs boson to photons and gluons, with $\bar{c}_{g}, \bar{c}_{\gamma}$ satisfying the naive estimates (2.9). For example, loops of stops lead to $\bar{c}_{g} \sim\left(g_{*}^{2} / 16 \pi^{2}\right)\left(m_{W}^{2} / m_{\tilde{t}}^{2}\right)$, where $g_{*}=y_{t}$ or $A_{t} / m_{\tilde{t}}$.

This situation has to be contrasted with the case of strongly coupled theories. There, our power counting (2.9) singles out $\bar{c}_{H}, \bar{c}_{u, d}$ as the dominant Wilson coefficients $\left(\bar{c}_{6}\right.$ controls only the Higgs self-interaction and measuring it at the LHC will be challenging), while $\bar{c}_{W}$ and $\bar{c}_{B}$ are suppressed by the ratio $\left(g / g_{*}\right)^{2}$. Furthermore, a composite Higgs boson can be naturally light if it is the pseudo Nambu-Goldstone boson associated to the dynamical breaking of a global symmetry of the strong dynamics. This implies that the coefficients $\bar{c}_{g}$ and $\bar{c}_{\gamma}$ will also be suppressed by a factor $\left(g_{G_{k}} / g_{*}\right)^{2}$, where $g_{G_{t}}$ is some weak spurion breaking the Goldstone symmetry. The modifications in the gluon-fusion production cross section and in the decay rate to photons are thus controlled by $\bar{c}_{H}$ and $\bar{c}_{u}$.

The harvest of data collected by the LHC certainly calls for a definite theoretical framework to describe the Higgs-like resonance and compute production and decay rates accurately in perturbation theory without restricting to the SM hypothesis. Effective Lagrangians are one of the tools at our disposal to achieve this goal. Elaborating on the operator classification of ref. [4], we estimated the present bounds on the Wilson coefficients and provided accurate expressions for the Higgs decay rates including various effects that were previously omitted in the literature. Assuming that the observed Higgs-like resonance is a spin- 0 and CP-even particle, we discussed two general formulations of the effective Lagrangian, one of which relies on the linear realization of $\mathrm{SU}(2)_{L} \times \mathrm{U}(1)_{Y}$ at high energies. One of the questions that can be addressed by considering these two parametrizations is whether the theory of New Physics flows to the SM in the infrared, that is, whether the Higgs-like resonance is part of an EW doublet. If all the Higgs signal strengths measured at the LHC converge towards the SM prediction, it would be a very suggestive indication that indeed the Higgs boson combines together with the longitudinal components of the $W$ and $Z$ to form an EW doublet, since any other alternative requires some tuning to fake the SM rates. On the other hand, the doublet nature of the Higgs boson would be less obvious to establish if the signal strengths exhibit deviations from their SM predictions (but note that some deviations in the signal strenghts could unambiguously indicate that the Higgs boson is not part of a doublet, this is in particular the case if a large breaking of the custiodial symmetry is observed in conflict with the strong bound already existing from EW precision data). We have pointed out that, if the EWSB dynamics is custodially symmetric, it is not possible to test whether the Higgs boson is part of a doublet by means of single-Higgs processes alone. A direct proof can come only from processes with multi-Higgs bosons in the final states [75], which are however challenging to study at the LHC. Precisely establishing the CP nature of the Higgs boson is another question that also requires accurate computations. If there is little doubt that the observed resonance has a large CP-even component, the possibility of a small mixing with a CP-odd component remains alive, and dedicated analyses will have to be performed to bound the mixing angle between the two components. To this aim too, an effective Lagrangian including the CP- 
odd operators listed in appendix C provides the theoretical framework where this question can be addressed quantitatively.

The absence so far of direct signals of New Physics at the LHC indicates that the road to unveil the origin of the electroweak symmetry breaking might be long and go through precision analyses rather than copious production of new particles. For such a task, the well established technology of effective field theories is the most powerful and general tool we have to analyze the Higgs data and put them into a coherent picture together with the existing experimental information without assuming the validity of the Standard Model. There is still time for the LHC to disprove this pessimistic eventuality by reporting the discovery of new light particles or large shifts in some of the Higgs couplings. It is clear, however, that if the New Physics continues to remain elusive, a precise investigation of the Higgs properties will become the most urgent programme in high-energy physics both for the experimental and the theoretical community.

\section{Acknowledgments}

We thank B. Gavela, A. De Rujula, J.R. Espinosa, A. Falkowski, E. Franco, L. Merlo, A. Pomarol, R. Rattazzi, F. Riva, L. Silvestrini, M. Trott for insightful discussions, and the participants of the LHC Higgs XS Working Group, in particular A. David, A. Denner, M. Dührssen, M. Grazzini, G. Passarino and G. Weiglein for discussions and comments. We also thank J.F. Kamenik for useful explanations on the results of ref. [41] and we thank A. Pomarol and E. Masso for pointing out a sign error in eq. (2.13) and we thank F. Maltoni for reminding us about the Bianchi identities. This research has been partly supported by the European Commission under the ERC Advanced Grant 226371 MassTeV and the contract PITN-GA-2009-237920 UNILHC. C.G. is supported by the Spanish Ministry MICNN under contract FPA2010-17747. The work of R.C. was partly supported by the ERC Advanced Grant No. 267985 Electroweak Symmetry Breaking, Flavour and Dark Matter: One Solution for Three Mysteries (DaMeSyFla). M.M. is supported by the DFG SFB/TR9 Computational Particle Physics.

\section{A SM Lagrangian: notations and conventions}

In this appendix, we collect the conventions used throughout this paper. The field content decomposes under $\mathrm{SU}(3)_{C} \times \mathrm{SU}(2)_{L} \times \mathrm{U}(1)_{Y}$ as

$$
\begin{aligned}
& H=(1,2,1 / 2), \quad L_{L}^{i}=(1,2,-1 / 2), \quad l_{R}^{i}=(1,1,-1), \\
& q_{L}^{i}=(3,2,1 / 6), \quad u_{R}^{i}=(3,1,2 / 3), \quad d_{R}^{i}=(3,1,-1 / 3),
\end{aligned}
$$

where the hypercharge is defined as $Y=Q-T_{3 L}$, and $i=1,2,3$ is a flavor index. The action of the gauge group is fully characterized by the conventions used to define the covariant derivative. For instance, for the left-handed quark doublet, we have

$$
D_{\mu} q_{L}=\left(\partial_{\mu}-\frac{i}{2} g_{S} \lambda^{a} g_{\mu}^{a}-\frac{i}{2} g \sigma^{i} W_{\mu}^{i}-\frac{i}{6} g^{\prime} B_{\mu}\right) q_{L}
$$


where $\lambda^{a}, a=1 \ldots 8$, and $\sigma^{i}, i=1 \ldots 3$, are the usual Gell-Mann and Pauli matrices. Accordingly, the gauge-field strengths are defined as

$$
G_{\mu \nu}^{a}=\partial_{\mu} g_{\nu}^{a}-\partial_{\nu} g_{\mu}^{a}+g_{S} f^{a b c} g_{\mu}^{b} g_{\nu}^{c}
$$

where $f^{a b c}$ are the $\mathrm{SU}(3)$ structure constants.

The Yukawa interactions of the up-type quarks involve the Higgs charge-conjugate doublet defined as

$$
H^{c}=i \sigma^{2} H^{*}
$$

The renormalizable Lagrangian of the SM thus reads:

$$
\begin{aligned}
\mathcal{L}_{S M}= & -\frac{1}{4} G_{\mu \nu}^{a} G^{a \mu \nu}-\frac{1}{4} W_{\mu \nu}^{i} W^{i \mu \nu}-\frac{1}{4} B_{\mu \nu} B^{\mu \nu}+\left(D^{\mu} H\right)^{\dagger}\left(D_{\mu} H\right) \\
& +i\left(\bar{L}_{L} \gamma^{\mu} D_{\mu} L_{L}+\bar{l}_{R} \gamma^{\mu} D_{\mu} l_{R}+\bar{q}_{L} \gamma^{\mu} D_{\mu} q_{L}+\bar{u}_{R} \gamma^{\mu} D_{\mu} u_{R}+\bar{d}_{R} \gamma^{\mu} D_{\mu} d_{R}\right) \\
& +\mu_{H}^{2} H^{\dagger} H-\lambda\left(H^{\dagger} H\right)^{2}+\left(y_{u} \bar{q}_{L} H^{c} u_{R}+y_{d} \bar{q}_{L} H d_{R}+y_{l} \bar{L}_{L} H l_{R}+\text { h.c. }\right)
\end{aligned}
$$

\section{B Electroweak Chiral Lagrangian in non-unitary gauge}

We report here the expression of the EW chiral Lagrangian valid in a generic gauge and in the most general case in which the $\mathrm{SU}(2)_{L} \times \mathrm{U}(1)_{Y}$ is non-linearly realized. For simplicity, we will restrict to the case in which the EWSB dynamics has a custodial invariance. The scalar $h$ is assumed to be CP-even and a singlet of the custodial symmetry, and does not necessarily belong to an $\mathrm{SU}(2)_{L}$ doublet. The Lagrangian can be expanded in terms with an increasing number of derivatives

$$
\mathcal{L}=\mathcal{L}_{0}+\mathcal{L}_{E W S B}, \quad \mathcal{L}_{E W S B}=-V(h)+\mathcal{L}^{(2)}+\mathcal{L}^{(4)}+\ldots
$$

where $\mathcal{L}_{0}$ contains the kinetic terms of the $\mathrm{SU}(3)_{c} \times \mathrm{SU}(2)_{L} \times \mathrm{U}(1)_{Y}$ gauge fields and of the SM fermions, $\mathcal{L}_{E W S B}$ describes the sector responsible for EWSB, and $V(h)$ is the potential for $h[51]$ :

$$
V(h)=\frac{1}{2} m_{h}^{2} h^{2}+c_{3} \frac{1}{6}\left(\frac{3 m_{h}^{2}}{v}\right) h^{3}+\ldots
$$

Under the request of $\mathrm{SU}(2)_{V}$ custodial symmetry, the longitudinal $W$ and $Z$ polarizations correspond to the $\mathrm{NG}$ bosons of the global coset $\mathrm{SU}(2)_{L} \times \mathrm{SU}(2)_{R} / \mathrm{SU}(2)_{V}$ and are described by the $2 \times 2$ matrix

$$
\Sigma(x)=\exp \left(i \sigma^{a} \chi^{a}(x) / v\right),
$$

where $\sigma^{a}$ are the Pauli matrices. $\mathrm{SU}(2)_{L} \times \mathrm{U}(1)_{Y}$ (local) transformations read as

$$
\Sigma(x) \rightarrow U_{L} \Sigma(x) U_{Y}^{\dagger}, \quad U_{L}=\exp \left(i \alpha_{L}^{a} \sigma^{a}\right), \quad U_{Y}=\exp \left(i \alpha_{Y} \sigma^{3}\right)
$$

and the covariant derivative is defined by

$$
D_{\mu} \Sigma=\partial_{\mu} \Sigma-\frac{i g}{2} W_{\mu}^{a} \sigma^{a} \Sigma+\frac{i g^{\prime}}{2} B_{\mu} \Sigma \sigma^{3} .
$$


At the level of two derivatives one has [51]:

$$
\begin{aligned}
\mathcal{L}^{(2)}= & \frac{1}{2}\left(\partial_{\mu} h\right)^{2}+\frac{v^{2}}{4} \operatorname{Tr}\left(D_{\mu} \Sigma^{\dagger} D^{\mu} \Sigma\right)\left(1+2 c_{V} \frac{h}{v}+\cdots\right) \\
& -\frac{v}{\sqrt{2}} \lambda_{i j}^{u}\left(\bar{u}_{L}^{(i)}, \bar{d}_{L}^{(i)}\right) \Sigma\left(u_{R}^{(i)}, 0\right)^{T}\left(1+c_{u} \frac{h}{v}+\cdots\right)+h . c . \\
& -\frac{v}{\sqrt{2}} \lambda_{i j}^{d}\left(\bar{u}_{L}^{(i)}, \bar{d}_{L}^{(i)}\right) \Sigma\left(0, d_{R}^{(i)}\right)^{T}\left(1+c_{d} \frac{h}{v}+\cdots\right)+h . c . \\
& -\frac{v}{\sqrt{2}} \lambda_{i j}^{l}\left(\bar{\nu}_{L}^{(i)}, \bar{l}_{L}^{(i)}\right) \Sigma\left(0, l_{R}^{(i)}\right)^{T}\left(1+c_{l} \frac{h}{v}+\cdots\right)+h . c .
\end{aligned}
$$

where the dots stand for terms with two or more Higgs fields and an implicit sum over flavor indices $i, j=1,2,3$ has been understood. After rotating to the fermion mass eigenbasis and by choosing the unitary gauge $\Sigma(x)=1$, the sum of (B.2) and (B.6) coincides with the first two lines of eq. (3.23) with $c_{W}=c_{Z}=c_{V}$.

At the level of four derivatives, there are 6 independent bosonic operators which affect cubic vertices with one $h$ field: ${ }^{24}$

$$
\begin{aligned}
\mathcal{L}^{(4)}= & c_{W W}^{\prime} W_{\mu \nu}^{a} W^{\mu \nu a} \frac{h}{v}+c_{W B}^{\prime} \operatorname{Tr}\left(\Sigma^{\dagger} W_{\mu \nu}^{a} \sigma^{a} \Sigma B_{\mu \nu} \sigma^{3}\right) \frac{h}{v}+c_{B B}^{\prime} B_{\mu \nu} B^{\mu \nu} \frac{h}{v} \\
& +\frac{c_{W}^{\prime}}{m_{W}} D^{\mu} W_{\mu \nu}^{a} \operatorname{Tr}\left(\Sigma^{\dagger} \sigma^{a} i \overleftrightarrow{D_{\nu}}{ }_{\nu}\right) h-\frac{c_{B}^{\prime}}{m_{W}} \partial^{\mu} B_{\mu \nu} \operatorname{Tr}\left(\Sigma^{\dagger} i \overleftrightarrow{D}_{\nu} \Sigma \sigma^{3}\right) h \\
& +\frac{c_{g g}}{2} G_{\mu \nu}^{a} G^{a \mu \nu} \frac{h}{v}+\ldots
\end{aligned}
$$

The dots stand for terms which have two or more $h$ fields or do not lead to cubic vertices, see refs. $[73,74]$ for the complete list of bosonic operators in $\mathcal{L}^{(4)}$. In the unitary gauge, eq. (B.8) coincides with the last three lines of eq. (3.23). More specifically, the coefficients $c_{W W}, c_{Z Z}, c_{Z \gamma}, c_{\gamma \gamma}$ can be written as linear combinations of $c_{W W}^{\prime}, c_{B B}^{\prime}, c_{W B}^{\prime}$,

$$
\begin{aligned}
c_{W W} & =2 c_{W W}^{\prime} \\
c_{Z Z} & =2\left(\cos ^{2} \theta_{W} c_{W W}^{\prime}-2 \sin \theta_{W} \cos \theta_{W} c_{W B}^{\prime}+\sin ^{2} \theta_{W} c_{B B}^{\prime}\right) \\
c_{\gamma \gamma} & =2\left(\sin ^{2} \theta_{W} c_{W W}^{\prime}+2 \sin \theta_{W} \cos \theta_{W} c_{W B}^{\prime}+\cos ^{2} \theta_{W} c_{B B}^{\prime}\right) \\
c_{Z \gamma} & =2\left(\sin \theta_{W} \cos \theta_{W} c_{W W}^{\prime}+\cos 2 \theta_{W} c_{W B}^{\prime}-\sin \theta_{W} \cos \theta_{W} c_{B B}^{\prime}\right),
\end{aligned}
$$

while $c_{W \partial W}, c_{Z \partial Z}$ can be expressed in terms of $c_{W}^{\prime}, c_{B}^{\prime}$ :

$$
\begin{aligned}
c_{W \partial W} & =4 c_{W}^{\prime} \\
c_{Z \partial Z} & =4 c_{W}^{\prime}+4 \tan \theta_{W} c_{B}^{\prime} \\
c_{Z \partial \gamma} & =4 \tan \theta_{W} c_{W}^{\prime}-4 c_{B}^{\prime} .
\end{aligned}
$$

\footnotetext{
${ }^{24}$ Another convenient basis, which can be more easily compared to eq. (3.23), is one in which the first two operators of eq. (B.8) are replaced by

$$
W_{\mu \nu}^{a} \operatorname{Tr}\left[\Sigma^{\dagger} \sigma^{a} i \overleftrightarrow{D}^{\mu} \Sigma\right] \partial^{\nu} h, \quad B_{\mu \nu} \operatorname{Tr}\left[\Sigma^{\dagger} i \overleftrightarrow{D^{\mu}} \Sigma \sigma^{3}\right] \partial^{\nu} h
$$

This is in fact the basis adopted in ref. [73]. 
Notice that eqs. (B.9) and (B.10) are directly implied by eq. (3.30), which follows from custodial invariance. It is simple to verify that the identities (3.24) and (3.25) are satisfied by the couplings appearing on the left-hand sides of respectively eq. (B.9) and (B.10).

The above discussion shows explicitly that every operator in eq. (3.23) can be dressed up with $\mathrm{NG}$ bosons and made manifestly invariant under local $\mathrm{SU}(2)_{L} \times \mathrm{U}(1)_{Y}$ transformations. ${ }^{25}$

The part of eq. (B.1) which does not depend on the Higgs field $h$ coincides with the non-linear chiral Lagrangian for $\mathrm{SU}(2)_{L} \times \mathrm{U}(1)_{Y}$ [100-102], in the limit of exact custodial symmetry. This latter assumption can be relaxed by specifying the sources of explicit breaking of the custodial symmetry, i.e. its spurions, in terms of which one can construct additional operators formally invariant under $\mathrm{SU}(2)_{L} \times \mathrm{U}(1)_{Y}$ local transformations. For example, the list of operators that follows in the case in which custodial invariance is broken by a field with the EW quantum numbers of hypercharge has been recently discussed in ref. [74]. Since the choice of quantum numbers of the spurions is model-dependent (and in fact the strongest effects are expected to arise from the breaking due to the top quark, rather than hypercharge), we do not report here any particular list of operators, and prefer to refer to the existing literature for further details.

\section{Relaxing the CP-even hypothesis}

If one relaxes the hypothesis that $h$ is CP-even, there are six extra dimension- 6 operators that need to be added to the effective Lagrangian (2.2):

$$
\begin{aligned}
\Delta \mathcal{L}_{C P}= & \frac{i \tilde{c}_{H W} g}{m_{W}^{2}}\left(D^{\mu} H\right)^{\dagger} \sigma^{i}\left(D^{\nu} H\right) \tilde{W}_{\mu \nu}^{i}+\frac{i \tilde{c}_{H B} g^{\prime}}{m_{W}^{2}}\left(D^{\mu} H\right)^{\dagger}\left(D^{\nu} H\right) \tilde{B}_{\mu \nu} \\
& +\frac{\tilde{c}_{\gamma} g^{\prime 2}}{m_{W}^{2}} H^{\dagger} H B_{\mu \nu} \tilde{B}^{\mu \nu}+\frac{\tilde{c}_{g} g_{S}^{2}}{m_{W}^{2}} H^{\dagger} H G_{\mu \nu}^{a} \tilde{G}^{a \mu \nu} \\
& +\frac{\tilde{c}_{3 W} g^{3}}{m_{W}^{2}} \epsilon^{i j k} W_{\mu}^{i \nu} W_{\nu}^{j \rho} \tilde{W}_{\rho}^{k \mu}+\frac{\tilde{c}_{3 G} g_{S}^{3}}{m_{W}^{2}} f^{a b c} G_{\mu}^{a \nu} G_{\nu}^{b \rho} \tilde{G}_{\rho}^{c \mu}
\end{aligned}
$$

where the dual field strengths are defined as $\tilde{F}_{\mu \nu}=\frac{1}{2} \epsilon_{\mu \nu \rho \sigma} F^{\rho \sigma}$ for $F=W, B, G(\epsilon$ is the totally antisymmetric tensor normalized to $\epsilon_{0123}=1$ ). Furthermore, the coefficients of the operators involving fermions will be in general complex numbers.

In the case of the effective chiral Lagrangian with $\mathrm{SU}(2)_{L} \times \mathrm{U}(1)_{Y}$ non-linearly realized, there are four additional operators, to be added to those of eq. (B.8), which can affect cubic vertices with one $h$ field:

$$
\begin{aligned}
\Delta \mathcal{L}_{C P}^{(4)}= & \tilde{c}_{W W}^{\prime} \tilde{W}_{\mu \nu}^{a} W^{\mu \nu a} \frac{h}{v}+\tilde{c}_{W B}^{\prime} \operatorname{Tr}\left[\Sigma^{\dagger} \tilde{W}_{\mu \nu}^{a} \sigma^{a} \Sigma B_{\mu \nu} \sigma^{3}\right] \frac{h}{v} \\
& +\tilde{c}_{B B}^{\prime} \tilde{B}_{\mu \nu} B^{\mu \nu} \frac{h}{v}+\frac{\tilde{c}_{g g}}{2} \tilde{G}_{\mu \nu}^{a} G^{a \mu \nu} \frac{h}{v} .
\end{aligned}
$$

\footnotetext{
${ }^{25}$ Notice that $h$ is invariant under $\mathrm{SU}(2)_{L} \times \mathrm{SU}(2)_{R}$ (hence $\left.\mathrm{SU}(2)_{L} \times \mathrm{U}(1)_{Y}\right)$ transformations. In the case in which $h$ belongs to an $\mathrm{SU}(2)_{L}$ doublet $H$, this follows from the fact that $h$ parametrizes the norm of the doublet: $H^{\dagger} H=(v+h)^{2} / 2$.
} 
In the unitary gauge, both Lagrangians $\Delta \mathcal{L}_{C P}$ and $\Delta \mathcal{L}_{C P}^{(4)}$ are matched onto:

$$
\begin{aligned}
\Delta \mathcal{L}_{C P}^{(4)}= & \left(\tilde{c}_{W W} W_{\mu \nu}^{+} \tilde{W}^{-\mu \nu}+\frac{\tilde{c}_{Z Z}}{2} Z_{\mu \nu} \tilde{Z}^{\mu \nu}+\tilde{c}_{Z \gamma} Z_{\mu \nu} \tilde{\gamma}^{\mu \nu}+\frac{\tilde{c}_{\gamma \gamma}}{2} \gamma_{\mu \nu} \tilde{\gamma}^{\mu \nu}+\frac{\tilde{c}_{g g}}{2} G_{\mu \nu}^{a} \tilde{G}^{a \mu \nu}\right) \frac{h}{v} \\
& +\ldots
\end{aligned}
$$

When the EW symmetry is linearly realized, the coefficients of eq. (C.3) are related to the Wilson coefficients of eq. (C.1) through the same relations reported in table 1 with the simple exchange $c_{i} \rightarrow \tilde{c}_{i}$ (and with $c_{W}=c_{B}=0$ ). In the non-linear case, $\tilde{c}_{W W}, \tilde{c}_{Z Z}, \tilde{c}_{\gamma \gamma}$ and $\tilde{c}_{Z \gamma}$ are given in terms of the Wilson coefficients of eq. (C.2) by relations identical to the ones of eq. (B.9) (with $c_{i} \rightarrow \tilde{c}_{i}$ and $c_{2}=0$ ). Notice that the Bianchi identities ensure that $D_{\mu} \tilde{V}^{\mu \nu}=0$ and therefore there are no CP-odd analog to the operators $O_{V \partial V}$.

Finally, it should also be noted that when the CP-invariance assumption in the Higgs sector is relaxed, the couplings $c_{u, d, l}$ are allowed to take some complex values.

\section{Current bounds on dimension-6 operators}

In this appendix we explain how we derived the bounds on the coefficients of the dimension6 operators reported in section 2.1. For a given observable we construct a likelihood for the coefficients $\bar{c}_{i}$ as follows:

$$
L\left(\bar{c}_{i}\right) \propto \exp \left[-\left(O_{S M}+\delta O\left(\bar{c}_{i}\right)-O_{\exp }\right)^{2} /\left(2 \Delta O_{\text {exp }}^{2}\right)\right],
$$

where $O_{\exp } \pm \Delta O_{\exp }$ is the experimental value of the observable, $O_{S M}$ denotes its SM prediction and $\delta O\left(\bar{c}_{i}\right)$ is the correction due to the effective operators. If several observables constrain the same coefficients $\bar{c}_{i}$, the global likelihood is constructed by multiplying those of each observable. We include the theoretical uncertainty on the SM prediction by integrating over a nuisance parameter whose distribution is appropriately chosen. We then quote the bound on a given coefficient by marginalizing over the remaining ones.

Let us consider for example the bounds of eqs. (2.14) and (2.15). To derive them we used the EW fit performed in ref. [36] by the GFitter collaboration, and constructed a likelihood for the various coefficients by computing their contributions to the $Z$-pole observables. For the latter, we used the SM predictions and experimental inputs reported in table 1 of ref. [36], treating the uncertainties on the SM predictions as normally distributed. We performed two separate fits: one on the coefficients of the operators involving the light quarks $(u, d, s)$, and one on those with charged leptons and heavy quarks $(c, b)$. We thus neglected, for simplicity, the correlations between these two sets of coefficients. The relevant observables in the first fit are $\Gamma_{\text {tot }}, \sigma_{\text {had }}$ and $R_{l}$. They depend on the Wilson coefficients only through the following linear combination:

$$
\begin{aligned}
l= & \left(-\frac{1}{4}+\frac{1}{3} \sin ^{2} \theta_{W}\right)\left(\bar{c}_{H q 1}-\bar{c}_{H q 1}^{\prime}\right)+\left(\frac{1}{4}-\frac{1}{6} \sin ^{2} \theta_{W}\right)\left(\bar{c}_{H q 1}+\bar{c}_{H q 1}^{\prime}+\bar{c}_{H q 2}+\bar{c}_{H q 2}^{\prime}\right) \\
& +\frac{1}{3} \sin ^{2} \theta_{W} \bar{c}_{H u}-\frac{1}{6} \sin ^{2} \theta_{W}\left(\bar{c}_{H d}+\bar{c}_{H s}\right),
\end{aligned}
$$


which with $95 \%$ probability is constrained to lie in the interval

$$
-0.63 \times 10^{-3}<l<1.2 \times 10^{-3} .
$$

Although there are no further observables at the $Z$-pole which can resolve the degeneracy implied by this result, we thought it useful to report the limits that one obtains from eq. (D.3) by turning on one coefficient at the time. These are the bounds reported in eq. (2.14).

The second fit, performed on the coefficients of the operators with leptons and heavy quarks, makes use of all the observables at the $Z$ pole and counts 7 unknowns, specifically: $x_{i}=\left\{\left(\bar{c}_{H q 2}-\bar{c}_{H q 2}^{\prime}\right), \bar{c}_{H c},\left(\bar{c}_{H q 3}+\bar{c}_{H q 3}^{\prime}\right), \bar{c}_{H b}, \bar{c}_{H l},\left(\bar{c}_{H L}+\bar{c}_{H L}^{\prime}\right),\left(\bar{c}_{H L}-\bar{c}_{H L}^{\prime}\right)\right\}$. For simplicity we assume lepton universality, and thus take the coefficients $\bar{c}_{H l}, \bar{c}_{H L}, \bar{c}_{H L}^{\prime}$ to be the same for all the leptonic generations. In terms of the above variables, the result of the fit is summarized by their central values $\bar{x}_{i}$, standard deviations $\sigma_{i}$ and by the correlation matrix $\rho_{i j}$ :

$$
\begin{aligned}
& \bar{c}_{H q 2}-\bar{c}_{H q 2}^{\prime}=(5.8 \pm 4.4) \times 10^{-3} \\
& \bar{c}_{H c}=(5.9 \pm 8.5) \times 10^{-3} \\
& \bar{c}_{H q 3}+\bar{c}_{H q 3}^{\prime}=(-3.1 \pm 2.7) \times 10^{-3} \\
& \bar{c}_{H b}=(-3.5 \pm 1.3) \times 10^{-2} \\
& \bar{c}_{H l}=(1.6 \pm 5.4) \times 10^{-4} \\
& \bar{c}_{H L}+\bar{c}_{H L}^{\prime}=(7.6 \pm 5.2) \times 10^{-4} \\
& \bar{c}_{H L}-\bar{c}_{H L}^{\prime}=(5.5 \pm 15) \times 10^{-4} \\
& \rho=\left(\begin{array}{ccccccc}
1.0 & 0.74 & -0.037 & -0.072 & 0.24 & -0.057 & -0.14 \\
0.74 & 1.0 & -0.078 & -0.085 & 0.11 & 0.15 & 0.030 \\
-0.037 & -0.078 & 1.0 & 0.85 & -0.40 & -0.21 & 0.068 \\
-0.072 & -0.085 & 0.85 & 1.0 & -0.40 & -0.33 & -0.0024 \\
0.24 & 0.11 & -0.40 & -0.40 & 1.0 & 0.11 & 0.28 \\
-0.057 & 0.15 & -0.21 & -0.33 & 0.11 & 1.0 & -0.35 \\
-0.14 & 0.030 & 0.068 & -0.0024 & 0.28 & -0.35 & 1.0
\end{array}\right)
\end{aligned}
$$

The limits of eq. (2.15) have been obtained by making use of the above formulas and marginalizing over all the coefficients except the one on which the bound is reported.

For the limits of eqs. (2.11) and (2.12) we have used the fit on $S$ and $T$ performed in ref. [36], by marginalizing on one parameter to extract the bound on the other.

To derive eq. (2.17) we have used the theoretical predictions of the EDM of the neutron and mercury given in ref. [39] in terms of the dipole moments of the quarks (see eqs. (2.12), (3.65) and (3.71) of ref. [39]), and the experimental results for these observables given respectively in ref. [103] and ref. [104]. We included the theoretical errors by assuming that they are uniformly distributed within the stated intervals. Only two linear combinations of 
the coefficients $\bar{c}_{i}$ can be constrained in this way, since two are the observables at disposal:

$$
\begin{aligned}
& l_{1}=-\frac{2 m_{d}}{m_{W}^{2}}\left[\operatorname{Im}\left(\bar{c}_{d G}\right)+1.3 \operatorname{Im}\left(\bar{c}_{d B}-\bar{c}_{d W}\right)\right]-\frac{m_{u}}{m_{W}^{2}}\left[\operatorname{Im}\left(\bar{c}_{u G}\right)-0.64 \operatorname{Im}\left(\bar{c}_{u B}+\bar{c}_{u W}\right)\right] \\
& l_{2}=-\frac{2 m_{u}}{m_{W}^{2}} \operatorname{Im}\left(\bar{c}_{u G}\right)+\frac{2 m_{d}}{m_{W}^{2}} \operatorname{Im}\left(\bar{c}_{d G}\right) .
\end{aligned}
$$

Using $m_{u}=2.3 \mathrm{MeV}$ and $m_{d}=4.8 \mathrm{MeV}$ we obtain, with $95 \%$ probability:

$$
\begin{aligned}
& -1.59 \times 10^{-12} \mathrm{GeV}^{-1}<l_{1}<1.78 \times 10^{-12} \mathrm{GeV}^{-1} \\
& -1.82 \times 10^{-12} \mathrm{GeV}^{-1}<l_{2}<1.37 \times 10^{-12} \mathrm{GeV}^{-1} .
\end{aligned}
$$

From the above result, by turning on one coefficient at the time, one obtains the limits given in eq. (2.17). The bound on $\operatorname{Im}\left(\bar{c}_{t G}\right)$ of eq. (2.18) has been similarly derived from the neutron and mercury EDMs by following ref. [41] and making use of the formulas given there.

The limits of eq. (2.22) have been obtained from the experimental measurements of the electron [45] and muon [43] anomalous magnetic moments and their SM predictions (taken respectively from ref. [46] and refs. [43, 44]). In this case we have included the theoretical errors by assuming that they are normally distributed. All the remaining bounds reported in section 2.1, namely those of eqs. (2.19)-(2.21) and eq. (2.23) have been obtained by simply translating into our notation the results given in the references quoted in the text.

Open Access. This article is distributed under the terms of the Creative Commons Attribution License which permits any use, distribution and reproduction in any medium, provided the original author(s) and source are credited.

\section{References}

[1] ATLAS collaboration, Observation of a new particle in the search for the Standard Model Higgs boson with the ATLAS detector at the LHC, Phys. Lett. B 716 (2012) 1 [arXiv:1207.7214] [INSPIRE].

[2] CMS collaboration, Observation of a new boson at a mass of $125 \mathrm{GeV}$ with the CMS experiment at the LHC, Phys. Lett. B 716 (2012) 30 [arXiv:1207.7235] [InSPIRE].

[3] S. Weinberg, The quantum theory of fields, Cambridge University Press, Cambridge, U.K. (1996).

[4] G. Giudice, C. Grojean, A. Pomarol and R. Rattazzi, The Strongly-Interacting Light Higgs, JHEP 06 (2007) 045 [hep-ph/0703164] [INSPIRE].

[5] D.B. Kaplan and H. Georgi, $\mathrm{SU}(2) \times \mathrm{U}(1)$ Breaking by Vacuum Misalignment, Phys. Lett. B 136 (1984) 183 [INSPIRE].

[6] S. Dimopoulos and J. Preskill, Massless composites with massive constituents, Nucl. Phys. B 199 (1982) 206 [INSPIRE].

[7] T. Banks, Constraints on $\mathrm{SU}(2) \times \mathrm{U}(1)$ breaking by vacuum misalignment, Nucl. Phys. $\mathrm{B}$ 243 (1984) 125 [inSPIRE].

[8] D.B. Kaplan, H. Georgi and S. Dimopoulos, Composite Higgs Scalars, Phys. Lett. B 136 (1984) 187 [INSPIRE]. 
[9] H. Georgi, D.B. Kaplan and P. Galison, Calculation of the composite Higgs mass, Phys. Lett. B 143 (1984) 152 [INSPIRE].

[10] H. Georgi and D.B. Kaplan, Composite Higgs and Custodial SU(2), Phys. Lett. B 145 (1984) 216 [INSPIRE].

[11] M.J. Dugan, H. Georgi and D.B. Kaplan, Anatomy of a Composite Higgs Model, Nucl. Phys. B 254 (1985) 299 [INSPIRE].

[12] R. Contino, Y. Nomura and A. Pomarol, Higgs as a holographic pseudoGoldstone boson, Nucl. Phys. B 671 (2003) 148 [hep-ph/0306259] [INSPIRE].

[13] K. Agashe, R. Contino and A. Pomarol, The Minimal composite Higgs model, Nucl. Phys. B 719 (2005) 165 [hep-ph/0412089] [INSPIRE].

[14] A. Djouadi, J. Kalinowski and M. Spira, HDECAY: A Program for Higgs boson decays in the standard model and its supersymmetric extension, Comput. Phys. Commun. 108 (1998) 56 [hep-ph/9704448] [INSPIRE].

[15] A. Djouadi, M. Muhlleitner and M. Spira, Decays of supersymmetric particles: The Program SUSY-HIT (SUspect-SdecaY-HDECAY-InTerface), Acta Phys. Polon. B 38 (2007) 635 [hep-ph/0609292] [INSPIRE].

[16] R. Contino, M. Ghezzi, C. Grojean, M.M. Muhlleitner and M. Spira, eHDECAY: an implementation of the Higgs effective Lagrangian into HDECAY, work in progress.

[17] C. Burges and H.J. Schnitzer, Virtual effects of excited quarks as probes of a possible new hadronic mass scale, Nucl. Phys. B 228 (1983) 464 [INSPIRE].

[18] C.N. Leung, S. Love and S. Rao, Low-Energy Manifestations of a New Interaction Scale: Operator Analysis, Z. Phys. C 31 (1986) 433 [InSPIRE].

[19] W. Buchmüller and D. Wyler, Effective Lagrangian Analysis of New Interactions and Flavor Conservation, Nucl. Phys. B 268 (1986) 621 [InSPIRE].

[20] R. Rattazzi, Anomalous interactions at the Z0 pole, Z. Phys. C 40 (1988) 605 [INSPIRE].

[21] B. Grzadkowski, Z. Hioki, K. Ohkuma and J. Wudka, Probing anomalous top quark couplings induced by dimension-six operators at photon colliders, Nucl. Phys. B 689 (2004) 108 [hep-ph/0310159] [INSPIRE].

[22] P.J. Fox, Z. Ligeti, M. Papucci, G. Perez and M.D. Schwartz, Deciphering top flavor violation at the LHC with B factories, Phys. Rev. D 78 (2008) 054008 [arXiv:0704.1482] [inSPIRE].

[23] J. Aguilar-Saavedra, A Minimal set of top anomalous couplings, Nucl. Phys. B 812 (2009) 181 [arXiv: 0811.3842] [INSPIRE].

[24] J. Aguilar-Saavedra, A Minimal set of top-Higgs anomalous couplings, Nucl. Phys. B 821 (2009) 215 [arXiv: 0904.2387] [INSPIRE].

[25] C. Grojean, W. Skiba and J. Terning, Disguising the oblique parameters, Phys. Rev. D 73 (2006) 075008 [hep-ph/0602154] [INSPIRE].

[26] B. Grzadkowski, M. Iskrzynski, M. Misiak and J. Rosiek, Dimension-Six Terms in the Standard Model Lagrangian, JHEP 10 (2010) 085 [arXiv: 1008.4884] [INSPIRE].

[27] R. Barbieri, A. Pomarol, R. Rattazzi and A. Strumia, Electroweak symmetry breaking after LEP-1 and LEP-2, Nucl. Phys. B 703 (2004) 127 [hep-ph/0405040] [INSPIRE]. 
[28] R. Barbieri, Electroweak precision tests : what do we learn?, CERN-TH-6659-92 (1992).

[29] M.E. Peskin and T. Takeuchi, Estimation of oblique electroweak corrections, Phys. Rev. D 46 (1992) 381 [INSPIRE].

[30] K. Agashe and R. Contino, Composite Higgs-Mediated FCNC, Phys. Rev. D 80 (2009) 075016 [arXiv:0906.1542] [INSPIRE].

[31] G. Isidori, Flavor physics and CP-violation, arXiv:1302.0661 [INSPIRE].

[32] G. Isidori, Y. Nir and G. Perez, Flavor Physics Constraints for Physics Beyond the Standard Model, Ann. Rev. Nucl. Part. Sci. 60 (2010) 355 [arXiv:1002.0900] [InSPIRE].

[33] P. Sikivie, L. Susskind, M.B. Voloshin and V.I. Zakharov, Isospin Breaking in Technicolor Models, Nucl. Phys. B 173 (1980) 189 [inSPIRE].

[34] G. Altarelli and R. Barbieri, Vacuum polarization effects of new physics on electroweak processes, Phys. Lett. B 253 (1991) 161 [INSPIRE].

[35] G. Altarelli, R. Barbieri and S. Jadach, Toward a model independent analysis of electroweak data, Nucl. Phys. B 369 (1992) 3 [Erratum ibid. B 376 (1992) 444] [INSPIRE].

[36] M. Baak et al., The Electroweak Fit of the Standard Model after the Discovery of a New Boson at the LHC, Eur. Phys. J. C 72 (2012) 2205 [arXiv:1209.2716] [INSPIRE].

[37] M. Redi and A. Weiler, Flavor and CP Invariant Composite Higgs Models, JHEP 11 (2011) 108 [arXiv: 1106.6357] [INSPIRE].

[38] N. Vignaroli, $\Delta F=1$ constraints on composite Higgs models with LR parity, Phys. Rev. D 86 (2012) 115011 [arXiv: 1204.0478] [INSPIRE].

[39] M. Pospelov and A. Ritz, Electric dipole moments as probes of new physics, Annals Phys. 318 (2005) 119 [hep-ph/0504231] [INSPIRE].

[40] P. Paradisi and D.M. Straub, The SUSY CP Problem and the MFV Principle, Phys. Lett. B 684 (2010) 147 [arXiv:0906.4551] [INSPIRE].

[41] J.F. Kamenik, M. Papucci and A. Weiler, Constraining the dipole moments of the top quark, Phys. Rev. D 85 (2012) 071501 [arXiv:1107.3143] [InSPIRE].

[42] J. Aguilar-Saavedra, N. Castro and A. Onofre, Constraints on the Wtb vertex from early LHC data, Phys. Rev. D 83 (2011) 117301 [arXiv:1105.0117] [InSPIRE].

[43] Particle Data Group collaboration, J. Beringer et al., Review of Particle Physics (RPP), Phys. Rev. D 86 (2012) 010001 [inSPIRE].

[44] M. Davier, A. Hoecker, B. Malaescu and Z. Zhang, Reevaluation of the Hadronic Contributions to the Muon g-2 and to alpha(MZ), Eur. Phys. J. C 71 (2011) 1515 [Erratum ibid. C 72 (2012) 1874] [arXiv:1010.4180] [INSPIRE].

[45] D. Hanneke, S.F. Hoogerheide and G. Gabrielse, Cavity Control of a Single-Electron Quantum Cyclotron: Measuring the Electron Magnetic Moment, arXiv:1009.4831 [INSPIRE].

[46] G. Giudice, P. Paradisi and M. Passera, Testing new physics with the electron g-2, JHEP 11 (2012) 113 [arXiv:1208.6583] [INSPIRE].

[47] Muon (G-2) collaboration, G. Bennett et al., An Improved Limit on the Muon Electric Dipole Moment, Phys. Rev. D 80 (2009) 052008 [arXiv:0811.1207] [INSPIRE]. 
[48] J. Hudson, D. Kara, I. Smallman, B. Sauer, M. Tarbutt and E. A. Hinds, Improved measurement of the shape of the electron, Nature 473 (2011) 493 [INSPIRE].

[49] D. Kara, I. Smallman, J. Hudson, B. Sauer, M. Tarbutt and E. A. Hinds, Measurement of the electron's electric dipole moment using YbF molecules: methods and data analysis, New J. Phys. 14 (2012) 103051 [arXiv: 1208.4507] [InSPIRE].

[50] R. Rattazzi, Effective Lagrangian for a strongly-interacting light Higgs, talk given at the workshop Physics at LHC: from Experiment to Theory, Princeton University, Princeton, U.S.A., 21-24 March 2007 [http://indico.cern.ch/getFile.py/access?contribId=29\& resId $=0$ \&materialId $=$ slides\&confId $=13975]$.

[51] R. Contino, C. Grojean, M. Moretti, F. Piccinini and R. Rattazzi, Strong Double Higgs Production at the LHC, JHEP 05 (2010) 089 [arXiv: 1002.1011] [INSPIRE].

[52] R. Contino, L. Da Rold and A. Pomarol, Light custodians in natural composite Higgs models, Phys. Rev. D 75 (2007) 055014 [hep-ph/0612048] [INSPIRE].

[53] S. Choi, . Miller, D.J., M. Muhlleitner and P. Zerwas, Identifying the Higgs spin and parity in decays to $Z$ pairs, Phys. Lett. B 553 (2003) 61 [hep-ph/0210077] [INSPIRE].

[54] A. De Rujula, J. Lykken, M. Pierini, C. Rogan and M. Spiropulu, Higgs look-alikes at the LHC, Phys. Rev. D 82 (2010) 013003 [arXiv:1001.5300] [InSPIRE].

[55] S. Bolognesi, Y. Gao, A.V. Gritsan, K. Melnikov, M. Schulze, N. V. Tran and A. Whitbeck, On the spin and parity of a single-produced resonance at the LHC, Phys. Rev. D 86 (2012) 095031 [arXiv: 1208.4018] [INSPIRE].

[56] A. Azatov, A. Falkowski, C. Grojean and E. Kuflik, Constraining the effective Higgs Lagrangian at higher order via $h \rightarrow V V$, work in progress.

[57] J.R. Ellis, M.K. Gaillard and D.V. Nanopoulos, A Phenomenological Profile of the Higgs Boson, Nucl. Phys. B 106 (1976) 292 [InSPIRE].

[58] M.A. Shifman, A. Vainshtein, M. Voloshin and V.I. Zakharov, Low-Energy Theorems for Higgs Boson Couplings to Photons, Sov. J. Nucl. Phys. 30 (1979) 711 [inSPIRE].

[59] B.A. Kniehl and M. Spira, Low-energy theorems in Higgs physics, Z. Phys. C 69 (1995) 77 [hep-ph/9505225] [INSPIRE].

[60] A. Falkowski, Pseudo-goldstone Higgs production via gluon fusion, Phys. Rev. D 77 (2008) 055018 [arXiv: 0711.0828] [INSPIRE].

[61] I. Low and A. Vichi, On the production of a composite Higgs boson, Phys. Rev. D 84 (2011) 045019 [arXiv: 1010.2753] [INSPIRE].

[62] A. Azatov and J. Galloway, Light Custodians and Higgs Physics in Composite Models, Phys. Rev. D 85 (2012) 055013 [arXiv:1110.5646] [inSPIRE].

[63] M. Gillioz, R. Grober, C. Grojean, M. Muhlleitner and E. Salvioni, Higgs Low-Energy Theorem (and its corrections) in Composite Models, JHEP 10 (2012) 004 [arXiv:1206.7120] [INSPIRE].

[64] M. Redi, Composite MFV and Beyond, Eur. Phys. J. C 72 (2012) 2030 [arXiv:1203.4220] [INSPIRE].

[65] R. Barbieri, D. Buttazzo, F. Sala, D.M. Straub and A. Tesi, A 125 GeV composite Higgs boson versus flavour and electroweak precision tests, JHEP 05 (2013) 069 [arXiv:1211.5085] [INSPIRE]. 
[66] C. Degrande, J.-M. Gerard, C. Grojean, F. Maltoni and G. Servant, Non-resonant New Physics in Top Pair Production at Hadron Colliders, JHEP 03 (2011) 125 [arXiv: 1010.6304] [INSPIRE].

[67] H. Hesari and M.M. Najafabadi, Probing the Anomalous Couplings of the Top Quark with Gluon at the LHC and Tevatron, arXiv:1207.0339 [INSPIRE].

[68] C. Englert, A. Freitas, M. Spira and P.M. Zerwas, Constraining the Intrinsic Structure of Top-Quarks, Phys. Lett. B 721 (2013) 261 [arXiv:1210. 2570] [INSPIRE].

[69] C. Degrande, J. Gerard, C. Grojean, F. Maltoni and G. Servant, Probing Top-Higgs Non-Standard Interactions at the LHC, JHEP 07 (2012) 036 [Erratum ibid. 1303 (2013) 032] [arXiv: 1205.1065] [INSPIRE].

[70] S.R. Coleman, J. Wess and B. Zumino, Structure of phenomenological Lagrangians. 1., Phys. Rev. 177 (1969) 2239 [InSPIRE].

[71] C.G. Callan Jr., S.R. Coleman, J. Wess and B. Zumino, Structure of phenomenological Lagrangians. 2., Phys. Rev. 177 (1969) 2247 [INSPIRE].

[72] C. Burgess and D. London, Light spin one particles imply gauge invariance, hep-ph/9203215 [INSPIRE].

[73] A. Azatov, R. Contino and J. Galloway, Model-Independent Bounds on a Light Higgs, JHEP 04 (2012) 127 [Erratum ibid. 1304 (2013) 140] [arXiv: 1202.3415] [INSPIRE].

[74] R. Alonso, M. Gavela, L. Merlo, S. Rigolin and J. Yepes, The Effective Chiral Lagrangian for a Light Dynamical 'Higgs', Phys. Lett. B 722 (2013) 330 [arXiv:1212.3305] [INSPIRE].

[75] R. Contino, C. Grojean, D. Pappadopulo, R. Rattazzi, A. Thamm, Strong Higgs interactions at a Linear Collider, work in progress.

[76] G. Passarino, NLO Inspired Effective Lagrangians for Higgs Physics, Nucl. Phys. B 868 (2013) 416 [arXiv: 1209.5538] [INSPIRE].

[77] S. Weinberg, Phenomenological Lagrangians, Physica A 96 (1979) 327 [InSPIRE].

[78] C. Grojean, E.E. Jenkins, A.V. Manohar and M. Trott, Renormalization Group Scaling of Higgs Operators and $\Gamma(h \rightarrow \gamma \gamma)$, JHEP 04 (2013) 016 [arXiv:1301.2588] [INSPIRE].

[79] J. Elias-Miro, J. Espinosa, E. Masso and A. Pomarol, Renormalization of dimension-six operators relevant for the Higgs decays $h \rightarrow \gamma \gamma, \gamma Z$, arXiv:1302.5661 [INSPIRE].

[80] R. Barbieri, B. Bellazzini, V.S. Rychkov and A. Varagnolo, The Higgs boson from an extended symmetry, Phys. Rev. D 76 (2007) 115008 [arXiv: 0706.0432] [INSPIRE].

[81] A. Pomarol and J. Serra, Top Quark Compositeness: Feasibility and Implications, Phys. Rev. D 78 (2008) 074026 [arXiv: 0806.3247] [InSPIRE].

[82] S.L. Adler, J.C. Collins and A. Duncan, Energy-Momentum-Tensor Trace Anomaly in Spin 1/2 Quantum Electrodynamics, Phys. Rev. D 15 (1977) 1712 [INSPIRE].

[83] J.C. Collins, A. Duncan and S.D. Joglekar, Trace and Dilatation Anomalies in Gauge Theories, Phys. Rev. D 16 (1977) 438 [inSPIRE].

[84] N. Nielsen, The Energy Momentum Tensor in a Nonabelian Quark Gluon Theory, Nucl. Phys. B 120 (1977) 212 [INSPIRE].

[85] M. Farina, C. Grojean, F. Maltoni, E. Salvioni and A. Thamm, Lifting degeneracies in Higgs couplings using single top production in association with a Higgs boson, JHEP 05 (2013) 022 [arXiv: 1211.3736] [INSPIRE]. 
[86] K. Chetyrkin, B.A. Kniehl and M. Steinhauser, Decoupling relations to $O\left(\alpha_{S}^{3}\right)$ and their connection to low-energy theorems, Nucl. Phys. B 510 (1998) 61 [hep-ph/9708255] [INSPIRE].

[87] M. Krämer, E. Laenen and M. Spira, Soft gluon radiation in Higgs boson production at the LHC, Nucl. Phys. B 511 (1998) 523 [hep-ph/9611272] [InSPIRE].

[88] Y. Schröder and M. Steinhauser, Four-loop decoupling relations for the strong coupling, JHEP 01 (2006) 051 [hep-ph/0512058] [INSPIRE].

[89] K. Chetyrkin, J.H. Kuhn and C. Sturm, QCD decoupling at four loops, Nucl. Phys. B 744 (2006) 121 [hep-ph/0512060] [INSPIRE].

[90] T. Inami, T. Kubota and Y. Okada, Effective gauge theory and the effect of heavy quarks in Higgs boson decays, Z. Phys. C 18 (1983) 69 [INSPIRE].

[91] A. Djouadi, M. Spira and P. Zerwas, Production of Higgs bosons in proton colliders: QCD corrections, Phys. Lett. B 264 (1991) 440 [INSPIRE].

[92] K. Chetyrkin, B.A. Kniehl and M. Steinhauser, Hadronic Higgs decay to order $\alpha_{S}^{4}$, Phys. Rev. Lett. 79 (1997) 353 [hep-ph/9705240] [INSPIRE].

[93] M. Spira, A. Djouadi, D. Graudenz and P. Zerwas, Higgs boson production at the LHC, Nucl. Phys. B 453 (1995) 17 [hep-ph/9504378] [INSPIRE].

[94] P. Baikov and K. Chetyrkin, Higgs Decay into Hadrons to Order $\alpha_{s}^{5}$, Phys. Rev. Lett. 97 (2006) 061803 [hep-ph/0604194] [INSPIRE].

[95] J.F. Gunion and H.E. Haber, The CP conserving two Higgs doublet model: The Approach to the decoupling limit, Phys. Rev. D 67 (2003) 075019 [hep-ph/0207010] [inSPIRE].

[96] L. Randall, Two Higgs Models for Large Tan Beta and Heavy Second Higgs, JHEP 02 (2008) 084 [arXiv:0711.4360] [INSPIRE].

[97] J. Gunion and H.E. Haber, Higgs Bosons in Supersymmetric Models. 1., Nucl. Phys. B 272 (1986) 1 [Erratum ibid. B 402 (1993) 567] [INSPIRE].

[98] K. Blum, R.T. D'Agnolo and J. Fan, Natural SUSY Predicts: Higgs Couplings, JHEP 01 (2013) 057 [arXiv: 1206.5303] [INSPIRE].

[99] A. Azatov and J. Galloway, Electroweak Symmetry Breaking and the Higgs Boson: Confronting Theories at Colliders, Int. J. Mod. Phys. A 28 (2013) 1330004 [arXiv:1212.1380] [INSPIRE].

[100] T. Appelquist and C.W. Bernard, Strongly Interacting Higgs Bosons, Phys. Rev. D 22 (1980) 200 [INSPIRE].

[101] A.C. Longhitano, Heavy Higgs Bosons in the Weinberg-Salam Model, Phys. Rev. D 22 (1980) 1166 [INSPIRE].

[102] A.C. Longhitano, Low-Energy Impact of a Heavy Higgs Boson Sector, Nucl. Phys. B 188 (1981) 118 [INSPIRE].

[103] C. Baker et al., An Improved experimental limit on the electric dipole moment of the neutron, Phys. Rev. Lett. 97 (2006) 131801 [hep-ex/0602020] [INSPIRE].

[104] W. Griffith, M. Swallows, T. Loftus, M. Romalis, B. Heckel and E. N. Fortson, Improved Limit on the Permanent Electric Dipole Moment of Hg-199, Phys. Rev. Lett. 102 (2009) 101601 [INSPIRE]. 